\title{
Study of $D_{J}$ meson decays to $D^{+} \pi^{-}, D^{0} \pi^{+}$and $D^{*+} \pi^{-}$final states in $p p$ collisions
}

\section{LHCh}

\section{The LHCb collaboration}

E-mail: antimo.palano@ba.infn.it

ABSTRACT: A study of $D^{+} \pi^{-}, D^{0} \pi^{+}$and $D^{*+} \pi^{-}$final states is performed using $p p$ collision data, corresponding to an integrated luminosity of $1.0 \mathrm{fb}^{-1}$, collected at a centre-of-mass energy of $7 \mathrm{TeV}$ with the $\mathrm{LHCb}$ detector. The $D_{1}(2420)^{0}$ resonance is observed in the $D^{*+} \pi^{-}$final state and the $D_{2}^{*}(2460)$ resonance is observed in the $D^{+} \pi^{-}, D^{0} \pi^{+}$and $D^{*+} \pi^{-}$ final states. For both resonances, their properties and spin-parity assignments are obtained. In addition, two natural parity and two unnatural parity resonances are observed in the mass region between 2500 and $2800 \mathrm{MeV}$. Further structures in the region around 3000 $\mathrm{MeV}$ are observed in all the $D^{*+} \pi^{-}, D^{+} \pi^{-}$and $D^{0} \pi^{+}$final states.

KEYwords: Spectroscopy, Hadron-Hadron Scattering, Charm physics

ArXiv EPrint: $1307.4556 \mathrm{v} 2$ 


\section{Contents}

1 Introduction 1

2 Detector 3

3 Event selection $\quad 3$

4 Mass spectra 4

5 Simulation $\quad 7$

6 Mass fit model $\quad 7$

7 Fit to the $D^{*+} \pi^{-}$mass spectrum $\quad 8$

8 Spin-parity analysis of the $D^{*+} \pi^{-}$system 10

9 Fit to the $D^{+} \pi^{-}$and $D^{0} \pi^{+}$mass spectra 11

10 Cross-checks and systematic uncertainties 17

$\begin{array}{ll}11 \text { Discussion and conclusions } & 18\end{array}$

The LHCb collaboration $\quad 22$

\section{Introduction}

Charm meson spectroscopy provides a powerful test of the quark model predictions of the Standard Model. Many charm meson states, predicted in the 1980s [1], have not yet been observed experimentally. The expected spectrum for the $c \bar{u}$ system is shown in figure 1 (the spectrum of the $c \bar{d}$ system is almost identical). The $J^{P}$ states having $P=(-1)^{J}$ and therefore $J^{P}=0^{+}, 1^{-}, 2^{+}, \ldots$ are called natural parity states and are labelled as $D^{*}$, while unnatural parity indicates the series $J^{P}=0^{-}, 1^{+}, 2^{-}, \ldots$ The low-mass spectrum of the $c \bar{u}$ system is comprised of the ground states (1S), the orbital excitations with angular momentum $L=1,2$ (1P, 1D), and the first radial excitations (2S). Apart from the ground states $\left(D, D^{*}\right)$, only two of the $1 \mathrm{P}$ states, $D_{1}(2420)$ and $D_{2}^{*}(2460)$ [2], are experimentally well established since they have relatively narrow widths $(\sim 30 \mathrm{MeV}) .{ }^{1}$ In contrast, the broad $L=1$ states, $D_{0}^{*}(2400)$ and $D_{1}^{\prime}(2430)$, have been established by the Belle and BaBar experiments in exclusive $B$ decays $[3,4]$.

\footnotetext{
${ }^{1}$ We work in units where $c=1$.
} 


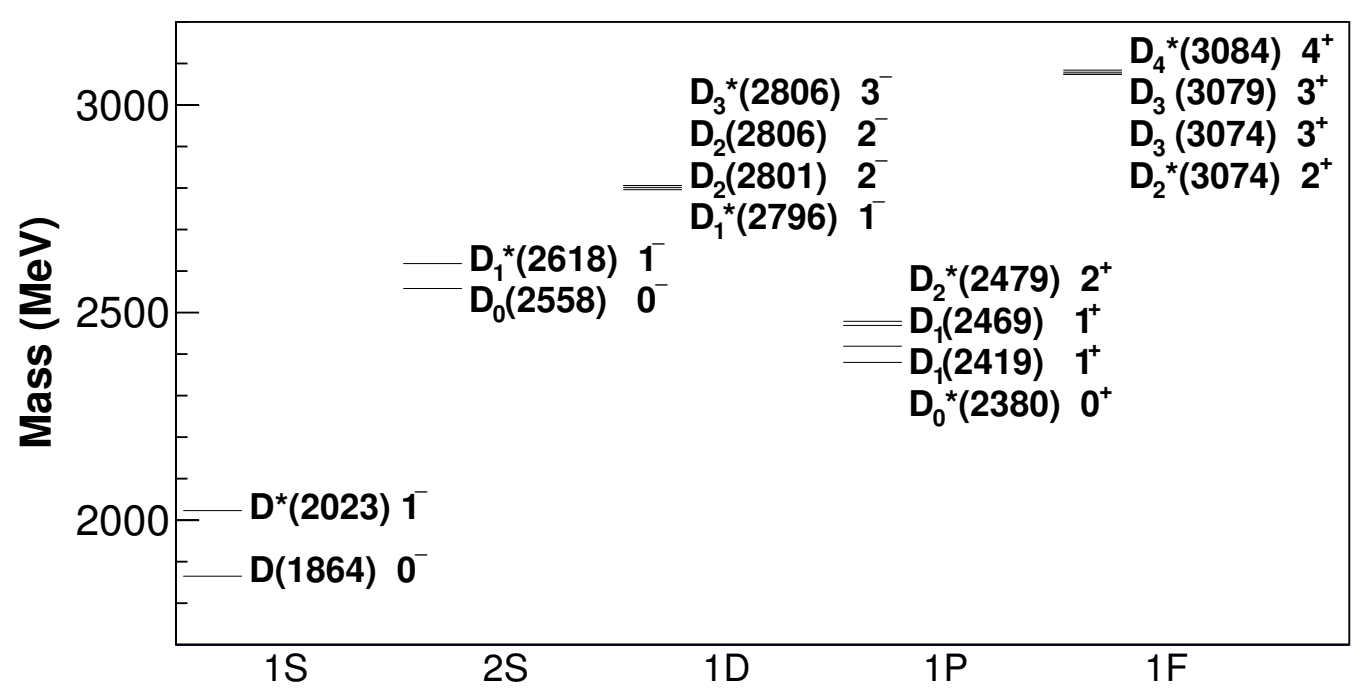

Figure 1. Modified Godfrey-Isgur mass predictions [1]. The figure shows the $c \bar{u}$ spectrum in which the masses have been scaled such that the ground state coincides with the $D^{0}$ mass. The $2^{-}$states, not shown in the original publication, have been inserted following the splitting structure of the $1 \mathrm{P}$ states.

The theoretical predictions are in agreement (within $20-30 \mathrm{MeV}$ ) with observations for the $1 \mathrm{~S}$ states and the $J^{P}=2^{+}$and $J^{P}=1^{+} 1 \mathrm{P}$ states. In the $c \bar{s}$ system, the $J^{P}=0^{+}$and $J^{P}=1^{+}$states (both $L=1$ ) have predicted masses about $100 \mathrm{MeV}$ higher than the measured masses of the $D_{s J}$ mesons. To quantitatively assess the accuracy of the quark model predictions, assumptions are needed to formulate a wave equation for quark-antiquark bound states starting from the QCD Lagrangian [5]. Nevertheless, the discrepancy between the predictions of various models and the mass measurements [6-9] suggests that some observed states in the $c \bar{s}$ case are not simple quark-antiquark configurations. Possible interpretations include more complex structures, such as bound states ("molecules") of other mesons [10], or mixtures of conventional quark-antiquark with four-quark components [11].

The properties of hadrons can be computed from the QCD Lagrangian using lattice calculations and the resulting $c \bar{u}, c \bar{d}$ and $c \bar{s}$ mass spectra can be compared to measurements. However, the calculation of hadronic quantities for dynamical light quarks is still a challenging task and different results are obtained [12-17].

To search for excited charmed mesons, labelled $D_{J}$, BaBar analyzed the inclusive production of the $D^{+} \pi^{-}, D^{0} \pi^{+}$and $D^{*+} \pi^{-}$final states in the inclusive reaction $e^{+} e^{-} \rightarrow c \bar{c} \rightarrow D^{(*)} \pi X$, where $X$ is any additional system [18]. ${ }^{2}$ They observe four signals, labelled $D(2550)^{0}, D^{*}(2600)^{0}, D(2750)^{0}$ and $D^{*}(2760)^{0}$, and the isospin partners $D^{*}(2600)^{+}$and $D^{*}(2760)^{+}$.

This paper reports a search for $D_{J}$ mesons in a data sample, corresponding to an integrated luminosity of $1.0 \mathrm{fb}^{-1}$, of $p p$ collisions collected at a centre-of-mass energy of $7 \mathrm{TeV}$ with the $\mathrm{LHCb}$ detector.

\footnotetext{
${ }^{2}$ Throughout the paper use of charge-conjugate decay modes is implied.
} 


\section{Detector}

The LHCb detector [19] is a single-arm forward spectrometer covering the pseudorapidity range $2<\eta<5$, designed for the study of particles containing $b$ or $c$ quarks. The detector includes a high precision tracking system consisting of a silicon-strip vertex detector surrounding the $p p$ interaction region, a large-area silicon-strip detector located upstream of a dipole magnet with a bending power of about $4 \mathrm{Tm}$, and three stations of silicon-strip detectors and straw drift tubes placed downstream. The combined tracking system has momentum resolution that varies from $0.4 \%$ at $5 \mathrm{GeV}$ to $0.6 \%$ at $100 \mathrm{GeV}$, and impact parameter resolution of $20 \mu \mathrm{m}$ for tracks with high transverse momentum $p_{\mathrm{T}}$ with respect to the beam direction. The impact parameter is defined as the perpendicular distance between the track path and the position of a $p p$ collision. Charged hadrons are identified using two ring-imaging Cherenkov (RICH) detectors. Photon, electron and hadron candidates are identified by a calorimeter system consisting of scintillating-pad and pre-shower detectors, an electromagnetic calorimeter and a hadronic calorimeter. Muons are identified by a system composed of alternating layers of iron and multiwire proportional chambers. The trigger [20] consists of a hardware stage, based on information from the calorimeter and muon systems, followed by a software stage which applies a full event reconstruction.

\section{Event selection}

The search for $D_{J}$ mesons is performed using the inclusive reactions

$$
p p \rightarrow D^{+} \pi^{-} X, p p \rightarrow D^{0} \pi^{+} X, p p \rightarrow D^{*+} \pi^{-} X,
$$

where $X$ represents a system composed of any collection of charged and neutral particles.

The charmed mesons in the final state are reconstructed in the decay modes $D^{+} \rightarrow K^{-} \pi^{+} \pi^{+}, D^{0} \rightarrow K^{-} \pi^{+}$and $D^{*+} \rightarrow D^{0} \pi^{+}$. Charged tracks are required to have good track fit quality, momentum $p>3 \mathrm{GeV}$ and $p_{\mathrm{T}}>250 \mathrm{MeV}$. These conditions are relaxed to lower limits for the pion originating directly from the $D^{*+}$ decay. Tracks pointing to a $p p$ collision vertex (primary vertex) are rejected by means of an impact parameter requirement in the reconstruction of the $D^{+}$and $D^{0}$ candidates. All tracks used to reconstruct the mesons must have a distance of closest approach to each other smaller than $0.5 \mathrm{~mm}$. The cosine of the angle between the momentum of the $D$ meson candidate and its direction, defined by the positions of the primary vertex and the meson decay vertex, is required to be larger than 0.99999 . This ensures that the $D$ meson candidates are produced at the primary vertex and reduces the contribution from particles originating from $b$-hadron decays. The reconstructed $D^{+}, D^{0}$ and $D^{*+}$ candidates are combined with all the right-sign charged pions in the event. Each of the $D^{+} \pi^{-}$, the $D^{0} \pi^{+}$, and the $D^{*+} \pi^{-}$ candidates are fitted to a common vertex with $\chi^{2} / \mathrm{ndf}<8$, where ndf is the number of degrees of freedom. The purity of the charmed meson candidates is enhanced by requiring the decay products to be identified by the RICH detectors, using the difference in the loglikelihood between the kaon and pion hypotheses $\Delta \ln \mathcal{L}_{K \pi}$ [21]. We require $\Delta \ln \mathcal{L}_{K \pi}>3$ for kaon tracks and a loose requirement of $\Delta \ln \mathcal{L}_{K \pi}<10$ for pions. In the reconstruction 
of $D^{+} \rightarrow K^{-} \pi^{+} \pi^{+}$decays, a small $D^{*+}$ signal in the $D^{0} \pi^{+}$mass spectrum is removed by demanding $\Delta m \equiv m\left(K^{-} \pi^{+} \pi^{+}\right)-m\left(K^{-} \pi^{+}\right)>152 \mathrm{MeV}$.

In order to reduce combinatorial background, the cosine of the angle between the momentum direction of the charged pion in the $D^{(*)} \pi^{ \pm}$rest frame and the momentum direction of the $D^{(*)} \pi^{ \pm}$system in the laboratory frame is required to be greater than zero. Due to the possible presence of multiple primary vertex candidates, it is required that the $D^{(*)}$ and the $\pi^{ \pm}$point to the same primary vertex.

To reduce any dependence on the mass scale, the invariant mass of the $D^{(*)} \pi^{ \pm}$system is calculated from the measured mass difference. For example, the $D^{0} \pi^{+}$invariant mass is given by

$$
m\left(D^{0} \pi^{+}\right)=m\left(K^{-} \pi^{+} \pi^{+}\right)-m\left(K^{-} \pi^{+}\right)+m_{D^{0}},
$$

where $m_{D^{0}}$ is the known value of the $D^{0}$ mass [2].

Figure 2 shows the $K^{-} \pi^{+} \pi^{+}, K^{-} \pi^{+}$and $D^{0} \pi^{+}$invariant mass spectra after the selection criteria are applied. The distributions are fitted by the sum of two Gaussian functions, with a common mean to describe the signal shape and a linear term to describe the combinatorial background. The mean mass resolutions for the three distributions are 8.1, 8.8 and $0.69 \mathrm{MeV}$, respectively. The signal regions, indicated by the dashed vertical lines, for the $D^{+}, D^{0}$ and $D^{*+}$ candidates correspond to $\pm 3 \sigma$ around the peak values and contain $15.1 \times 10^{6}, 20.4 \times 10^{6}$ and $6.4 \times 10^{6}$ candidates for the $D^{+} \pi^{-}, D^{0} \pi^{+}$and $D^{*+} \pi^{-}$modes, respectively.

\section{Mass spectra}

The $D^{+} \pi^{-}, D^{0} \pi^{+}$and $D^{*+} \pi^{-}$mass spectra are shown in figure 3. The $D^{+} \pi^{-}$and $D^{0} \pi^{+}$ mass spectra evidence strong $D_{2}^{*}(2460)$ signals, while in the $D^{*+} \pi^{-}$mass spectrum clear $D_{1}(2420)^{0}$ and $D_{2}^{*}(2460)^{0}$ signals are visible. A further reduction of the combinatorial background is achieved by performing an optimization of the signal significance and purity as a function of $p_{\mathrm{T}}$ of the $D^{(*)} \pi^{ \pm}$system using the well known $D_{1}(2420)$ and $D_{2}^{*}(2460)$ resonances. ${ }^{3}$ For this purpose, we fit the three mass spectra as explained in section 7 and section 9 and obtain, for each resonance, the signal yield $N_{\mathrm{S}}$ and background yield $N_{\mathrm{B}}$ events. We compute the signal significance $S=N_{\mathrm{S}} / \sqrt{N_{\mathrm{S}}+N_{\mathrm{B}}}$ and signal purity $P=N_{\mathrm{S}} /\left(N_{\mathrm{S}}+N_{\mathrm{B}}\right)$ and find that the requirement $p_{\mathrm{T}}\left(D^{(*)} \pi\right)>7.5 \mathrm{GeV}$ provides a good compromise between significance and purity. After the optimization $7.9 \times 10^{6}, 7.5 \times 10^{6}$ and $2.1 \times 10^{6} D^{+} \pi^{-}, D^{0} \pi^{+}$and $D^{*+} \pi^{-}$candidates are obtained. We also study the dependence of the signal to background ratio in the three mass spectra on the pseudorapidity of the $D^{(*)} \pi$ system and find a very weak correlation. We analyze, for comparison and using the same selections, the wrong-sign $D^{+} \pi^{+}, D^{0} \pi^{-}$and $D^{*+} \pi^{+}$combinations which are also shown in figure 3.

The $D^{+} \pi^{-}$mass spectrum, figure $3(\mathrm{a})$, shows a double peak structure around $2300 \mathrm{MeV}$ due to cross-feed from the decay

$$
D_{1}(2420)^{0} \text { or } D_{2}^{*}(2460)^{0} \rightarrow \pi^{-} D^{*+}\left(\rightarrow D^{+} \pi^{0} / \gamma\right)(32.3 \%),
$$

\footnotetext{
${ }^{3}$ We use the generic notation $D$ to indicate both neutral and charged $D$ mesons.
} 

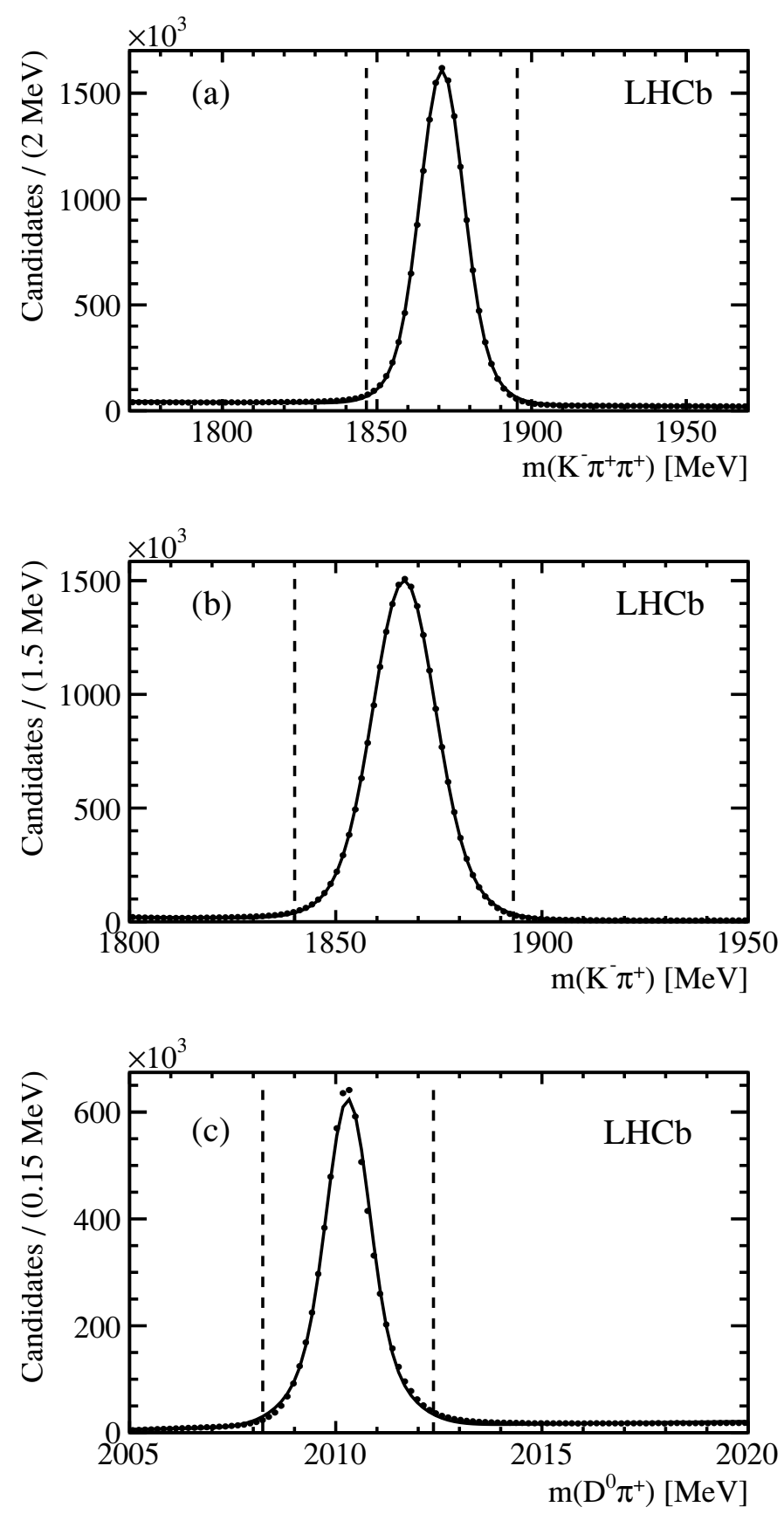

Figure 2. Invariant mass distribution for (a) $D^{+} \rightarrow K^{-} \pi^{+} \pi^{+}$, (b) $D^{0} \rightarrow K^{-} \pi^{+}$, and (c) $D^{*+} \rightarrow$ $D^{0} \pi^{+}$decays. The solid lines are the results from the fits described in the text. The vertical dashed lines indicate the signal regions.

where the $\pi^{0} / \gamma$ is not reconstructed; the last number, in parentheses, indicates the branching fraction of $D^{*+} \rightarrow D^{+} \pi^{0} / \gamma$ decays [2]. We observe a strong $D_{2}^{*}(2460)^{0}$ signal and weak structures around 2600 and $2750 \mathrm{MeV}$. The wrong-sign $D^{+} \pi^{+}$mass spectrum does not show any structure. 

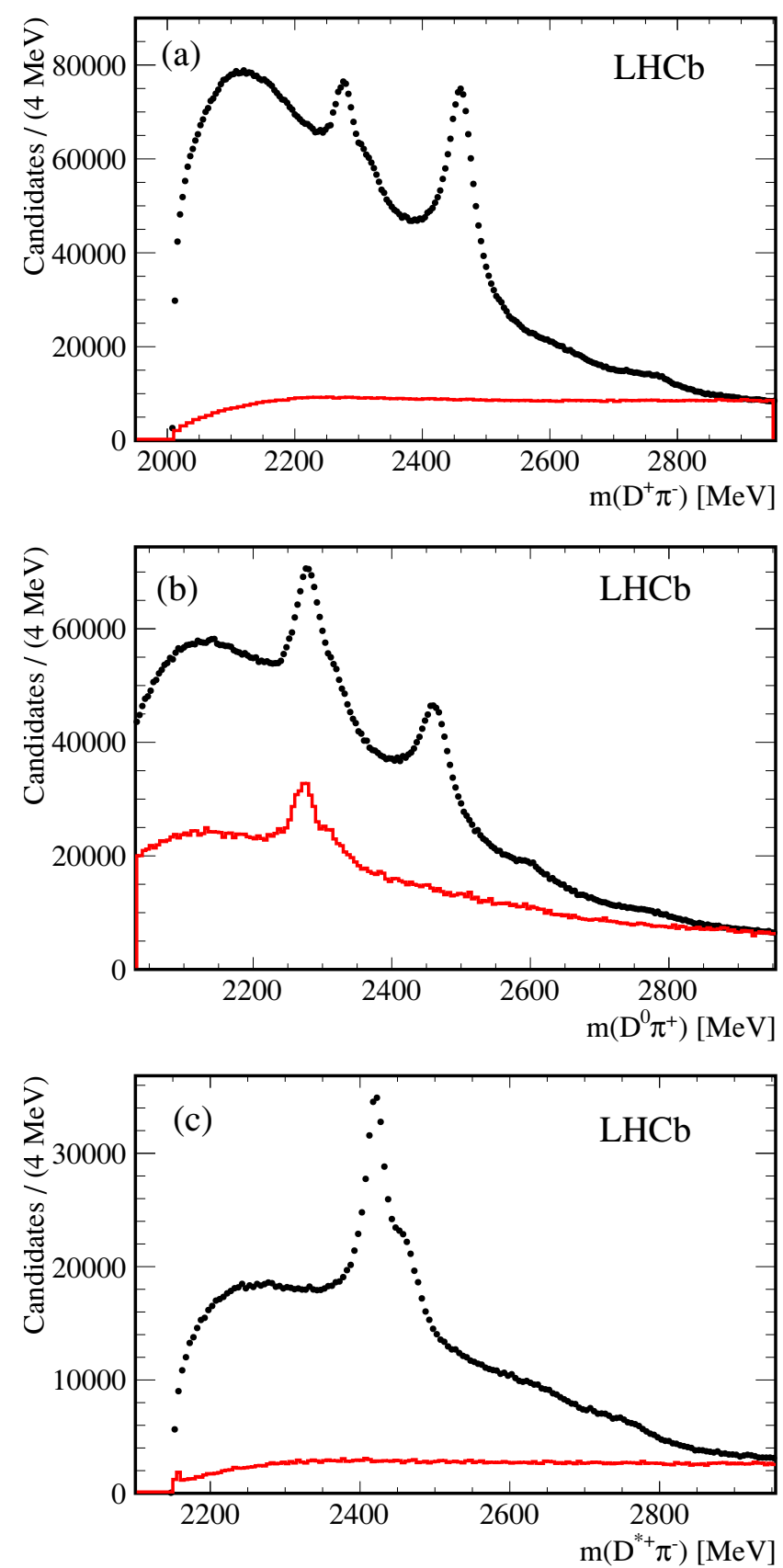

Figure 3. Invariant mass distribution for (a) $D^{+} \pi^{-}$, (b) $D^{0} \pi^{+}$and (c) $D^{*+} \pi^{-}$candidates (points). The full line histograms (in red) show the wrong-sign mass spectra for (a) $D^{+} \pi^{+}$, (b) $D^{0} \pi^{-}$and (c) $D^{*+} \pi^{+}$normalized to the same yield at high $D^{(*)} \pi$ masses.

The $D^{0} \pi^{+}$mass spectrum, figure 3(b), shows an enhanced double peak structure around $2300 \mathrm{MeV}$ due to cross-feed from the decays

$$
\begin{aligned}
D_{1}(2420)^{+} \text {or } D_{2}^{*}(2460)^{+} \rightarrow \pi^{+} D^{* 0} & \left(\rightarrow D^{0} \pi^{0}\right)(61.9 \%) \\
& \left(\rightarrow D^{0} \gamma\right)(38.1 \%)
\end{aligned}
$$


The $D_{2}^{*}(2460)^{+}$signal and weak structures around 2600 and $2750 \mathrm{MeV}$ are observed. In comparison, the wrong-sign $D^{0} \pi^{-}$mass spectrum does show the presence of structures in the $2300 \mathrm{MeV}$ mass region, similar to those observed in the $D^{0} \pi^{+}$mass spectrum. These structures are due to cross-feed from the decay

$$
D_{1}(2420)^{0} \text { or } D_{2}^{*}(2460)^{0} \rightarrow \pi^{-} D^{*+}\left(\rightarrow D^{0} \pi^{+}\right)(67.7 \%) .
$$

The $D^{*+} \pi^{-}$mass spectrum, figure $3(\mathrm{c})$, is dominated by the presence of the $D_{1}(2420)^{0}$ and $D_{2}^{*}(2460)^{0}$ signals. At higher mass, complex broad structures are evident in the mass region between 2500 and $2800 \mathrm{MeV}$.

\section{Simulation}

Simulated events are used to study the effects of the detector on the observed mass resolution. The $p p$ collisions are generated using Pyтнia 6.4 [22] with a specific LHCb configuration [23]. Decays of hadronic particles are described by EvTGen [24] and the interaction of the generated particles with the detector and its response are implemented using the GEANT4 toolkit [25, 26] as described in ref. [27].

Simulated events are reconstructed in the same manner as data. We analyze samples of full detector simulations to estimate the reconstruction efficiency, mass resolution and possible bias in the reconstruction chain. We also make use of simple event generator level simulations [28] to study kinematic effects. The tight trigger conditions and selection criteria have the effect of producing very low integrated efficiencies, which we calculate to be $(0.149 \pm 0.004) \%,(0.056 \pm 0.005) \%$ and $(0.064 \pm 0.003) \%$ for $D^{+} \pi^{-}, D^{0} \pi^{+}$and $D^{*+} \pi^{-}$ candidates, respectively.

To estimate the detector resolution we compare generated and reconstructed invariant masses and obtain experimental resolutions as functions of the reconstructed mass. The analysis of these simulated samples shows no bias in the reconstructed invariant masses. We estimate resolutions which, in the mass region between 2000 and $2900 \mathrm{MeV}$, are similar for the three mass spectra and range from 1.0 to $4.5 \mathrm{MeV}$ as a function of the mass. Since the widths of the resonances appearing in the three mass spectra are much larger than the experimental resolutions, resolution effects are neglected.

\section{Mass fit model}

Binned $\chi^{2}$ fits to the three mass spectra are performed. The $D_{2}^{*}(2460)$ and $D_{0}^{*}(2400)$ signal shapes in two-body decays are parameterized with a relativistic Breit-Wigner that includes the mass-dependent factors for a $\mathrm{D}$-wave and S-wave decay, respectively. The radius entering in the Blatt-Weisskopf [29] form factor is fixed to $4 \mathrm{GeV}^{-1}$. Other resonances appearing in the mass spectra are described by Breit-Wigner lineshapes. All Breit-Wigner expressions are multiplied by two-body phase space. The cross-feed lineshapes from $D_{1}(2420)$ and $D_{2}^{*}(2460)$ appearing in the $D^{+} \pi^{-}$and $D^{0} \pi^{+}$mass spectra are described by a Breit-Wigner function fitted to the data with the parameters given in table 1. Resonances are included 


\begin{tabular}{|llrr|}
\hline Final state & Parameter $(\mathrm{MeV})$ & $D_{1}(2420)$ & $D_{2}^{*}(2460)$ \\
\hline$D^{+} \pi^{-}$ & Mass & 2276.5 & 2319.8 \\
& Width & 38.3 & 50.0 \\
\hline$D^{0} \pi^{+}$ & Mass & 2278.4 & 2319.4 \\
& Width & 44.9 & 49.1 \\
\hline
\end{tabular}

Table 1. Breit-Wigner parameters describing the cross-feed from $D_{1}(2420)$ and $D_{2}^{*}(2460)$ in the $D^{+} \pi^{-}$and $D^{0} \pi^{+}$final states.

sequentially in order to test the $\chi^{2}$ improvement when a new contribution is included. The background $B(m)$ is described by an empirical shape [18]

$$
\begin{array}{ll}
B(m)=P(m) e^{a_{1} m+a_{2} m^{2}} & \text { for } m<m_{0}, \\
B(m)=P(m) e^{b_{0}+b_{1} m+b_{2} m^{2}} & \text { for } m>m_{0},
\end{array}
$$

where $P(m)$ is the two-body phase space and $m_{0}$ is a free parameter.

The two functions and their first derivatives are required to be continuous at $m_{0}$ such that

$$
\begin{aligned}
& b_{1}=a_{1}+2 m_{0}\left(a_{2}-b_{2}\right), \\
& b_{0}=m_{0}\left(a_{1}-b_{1}\right)+m_{0}^{2}\left(a_{2}-b_{2}\right) .
\end{aligned}
$$

Therefore the background model has four free parameters: $m_{0}, a_{1}, a_{2}$ and $b_{2}$.

\section{$7 \quad$ Fit to the $D^{*+} \pi^{-}$mass spectrum}

Due to the three-body decay and the availability of the helicity angle information, the fit to the $D^{*+} \pi^{-}$mass spectrum allows a spin analysis of the produced resonances and a separation of the different spin-parity components. We define the helicity angle $\theta_{\mathrm{H}}$ as the angle between the $\pi^{-}$and the $\pi^{+}$from the $D^{*+}$ decay, in the rest frame of the $D^{*+} \pi^{-}$ system. Full detector simulations are used to measure the efficiency as a function of $\theta_{\mathrm{H}}$, which is found to be uniform.

It is expected that the angular distributions are proportional to $\sin ^{2} \theta_{\mathrm{H}}$ for natural parity resonances and proportional to $1+h \cos ^{2} \theta_{\mathrm{H}}$ for unnatural parity resonances, where $h>0$ is a free parameter. The $D^{*} \pi$ decay of a $J^{P}=0^{+}$resonance is forbidden. Therefore candidates selected in different ranges of $\cos \theta_{\mathrm{H}}$ can enhance or suppress the different spin-parity contributions. We separate the $D^{*+} \pi^{-}$data into three different categories, summarized in table 2. The candidate yields for these categories are given in table 3 , which also reports the mass intervals, the number of bins, and the resulting $\chi^{2} /$ ndf in the fits to the different mass spectra.

The data and fit for the $D^{*+} \pi^{-}$enhanced unnatural parity sample are shown in figure 4 and the resulting fit parameters are summarized in table 4 . The mass spectrum is dominated by the presence of the unnatural parity $D_{1}(2420)^{0}$ resonance. The fitted natural parity $D_{2}^{*}(2460)^{0}$ contribution is consistent with zero, as expected. To obtain a good 


\begin{tabular}{|llc|}
\hline Category & Selection & natural parity fraction (\%) \\
\hline Enhanced unnatural parity sample & $\left|\cos \theta_{\mathrm{H}}\right|>0.75$ & 8.6 \\
Natural parity sample & $\left|\cos \theta_{\mathrm{H}}\right|<0.5$ & 68.8 \\
Unnatural parity sample & $\left|\cos \theta_{\mathrm{H}}\right|>0.5$ & 31.2 \\
\hline
\end{tabular}

Table 2. Definition of the categories selected by different ranges of $\cos \theta_{\mathrm{H}}$, and fraction of the total natural parity contribution.

\begin{tabular}{|llcccc|}
\hline Final state & Selection & $\begin{array}{c}\text { Fit Range } \\
(\mathrm{MeV})\end{array}$ & $\begin{array}{c}\text { Number } \\
\text { of bins }\end{array}$ & $\begin{array}{c}\text { Candidates } \\
\left(\times 10^{6}\right)\end{array}$ & $\chi^{2} / \mathrm{ndf}$ \\
\hline$D^{+} \pi^{-}$ & Total & $2050-3170$ & 280 & 7.90 & $551 / 261$ \\
\hline$D^{0} \pi^{+}$ & Total & $2050-3170$ & 280 & 7.50 & $351 / 262$ \\
\hline$D^{*+} \pi^{-}$ & Total & $2180-3170$ & 247 & 2.04 & $438 / 234$ \\
$D^{*+} \pi^{-}$ & Natural & & & 0.98 & $263 / 229$ \\
& $\begin{array}{l}\text { parity sample } \\
D^{*+} \pi^{-}\end{array}$ & & & 1.06 & $364 / 234$ \\
$D^{*+} \pi^{-}$ & $\begin{array}{l}\text { Unnatural } \\
\text { parity sample } \\
\text { Enhanced unnatural parity } \\
\text { sample }\end{array}$ & & & 0.55 & $317 / 230$ \\
\hline
\end{tabular}

Table 3. Mass intervals, number of bins, yields, and $\chi^{2} /$ ndf in the fits to the different mass spectra.

fit to the mass spectrum, three further resonances are needed. We label them $D_{J}(2580)^{0}$, $D_{J}(2740)^{0}$, and $D_{J}(3000)^{0}$. The presence of these states in this sample indicates unnatural parity assignments.

The masses and widths of the unnatural parity resonances are fixed in the fit to the natural parity sample. The fit is shown in figure 5 and the obtained resonance parameters are summarized in table 4 . The mass spectrum shows that the unnatural parity resonance $D_{1}(2420)^{0}$ is suppressed with respect to that observed in the enhanced unnatural parity sample. There is a strong contribution of the natural parity $D_{2}^{*}(2460)^{0}$ resonance and contributions from the $D_{J}(2580)^{0}, D_{J}(2740)^{0}$ and $D_{J}(3000)^{0}$ states. To obtain a good fit, two additional resonances are needed, which we label $D_{J}^{*}(2650)^{0}$ and $D_{J}^{*}(2760)^{0}$.

The unnatural parity sample is used as a cross-check. In this fit, the parameters of all the resonances are fixed to the values obtained from the previous fits. The fit is shown in figure 6 . We observe, as expected, small contributions from the natural parity resonances. We also fit the total $D^{*+} \pi^{-}$mass spectrum, again with all the resonance parameters fixed. The data and fit are shown in figure 7 .

Table 4 summarizes the measured resonance parameters and yields. The resonance parameters are obtained from the fits to the enhanced unnatural parity sample and natural parity sample, apart for the parameters of the $D_{1}(2420)^{0}$ resonance, which are extracted from the fit to the total sample. The significances are computed as $\sqrt{\Delta \chi^{2}}$ where $\Delta \chi^{2}$ is the difference between the $\chi^{2}$ values when a resonance is included or excluded from the fit while all the other resonances parameters are allowed to vary. All the statistical significances are well above $5 \sigma$. 


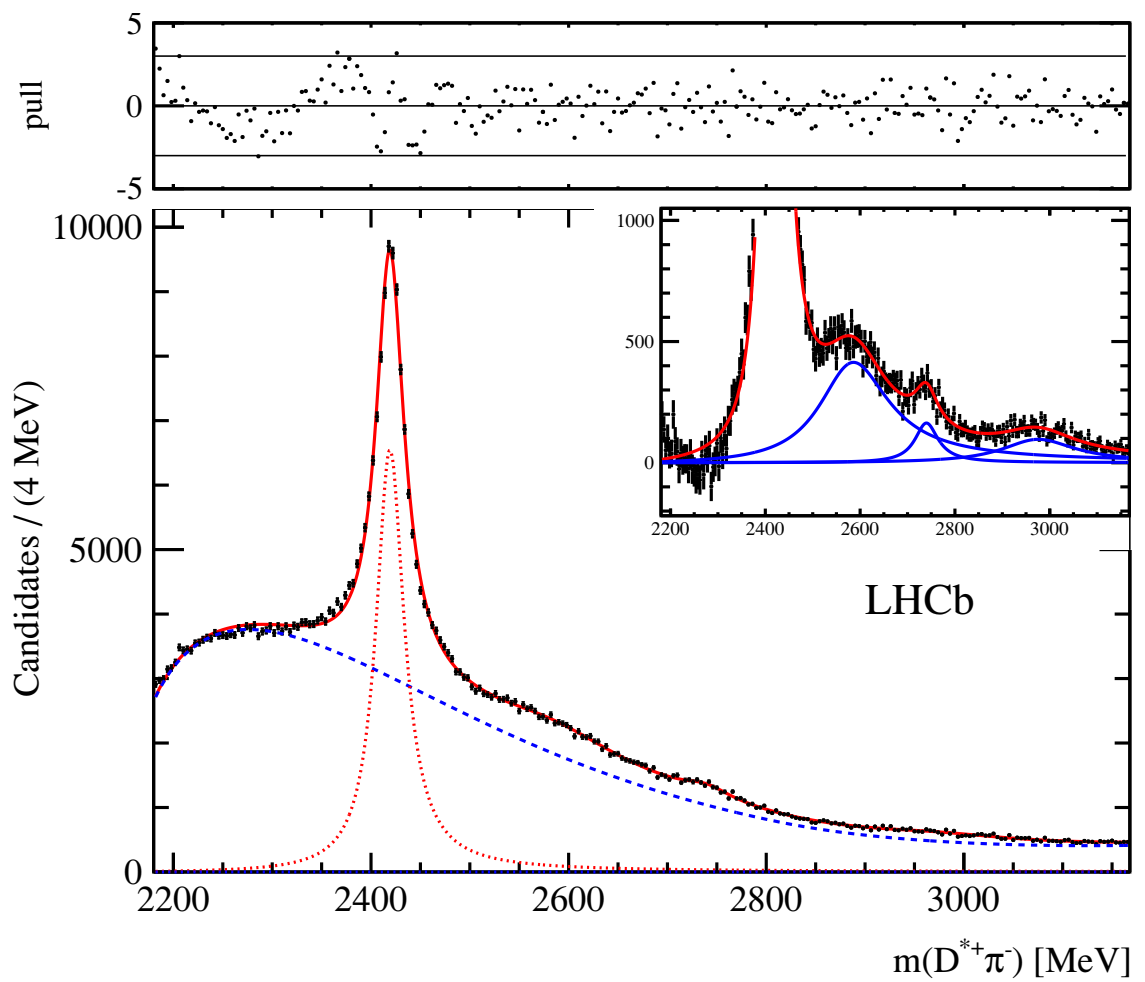

Figure 4. Fit to the $D^{*+} \pi^{-}$mass spectrum, enhanced unnatural parity sample, as defined in table 2. The dashed (blue) line shows the fitted background, the dotted (red) line the $D_{1}(2420)^{0}$ contribution. The inset displays the $D^{*+} \pi^{-}$mass spectrum after subtracting the fitted background. The full line curves (blue) show the contributions from $D_{J}(2580)^{0}, D_{J}(2740)^{0}$, and $D_{J}(3000)^{0}$ states. The top window displays the pull distribution where the horizontal lines indicate $\pm 3 \sigma$. The pull is defined as $\left(N_{\text {data }}-N_{\text {fit }}\right) / \sqrt{N_{\text {data }}}$.

\section{Spin-parity analysis of the $D^{*+} \pi^{-}$system}

In order to obtain information on the spin-parity assignment of the states observed in the $D^{*+} \pi^{-}$mass spectrum, the data are subdivided into ten equally spaced bins in $\cos \theta_{\mathrm{H}}$. The ten mass spectra are then fitted with the model described above with fixed resonance parameters to obtain the yields as functions of $\cos \theta_{\mathrm{H}}$ for each resonance.

The resulting distributions for $D_{1}(2420)^{0}$ and $D_{2}^{*}(2460)^{0}$ are shown in figure 8. They have been fitted using the functions described in table 5. A good description of the data is obtained in terms of the expected angular distributions for $J^{P}=1^{+}$and $J^{P}=2^{+}$ resonances.

Figure 9 shows the resulting distributions for the $D_{J}^{*}(2650)^{0}$ and $D_{J}^{*}(2760)^{0}$ states. In this case we compare the distributions with expectations from natural parity, unnatural parity and $J^{P}=0^{-}$. In the case of unnatural parity, the $h$ parameter, in $1+h \cos ^{2} \theta_{\mathrm{H}}$, is constrained to be positive and therefore the fit gives $h=0$. In both cases, the distributions are best fitted by the natural parity hypothesis.

Figure 10 shows the angular distributions for the $D_{J}(2580)^{0}, D_{J}(2740)^{0}$ and $D_{J}(3000)^{0}$ states. The distributions are fitted with natural parity and unnatural parity. The $J^{P}=0^{-}$ 


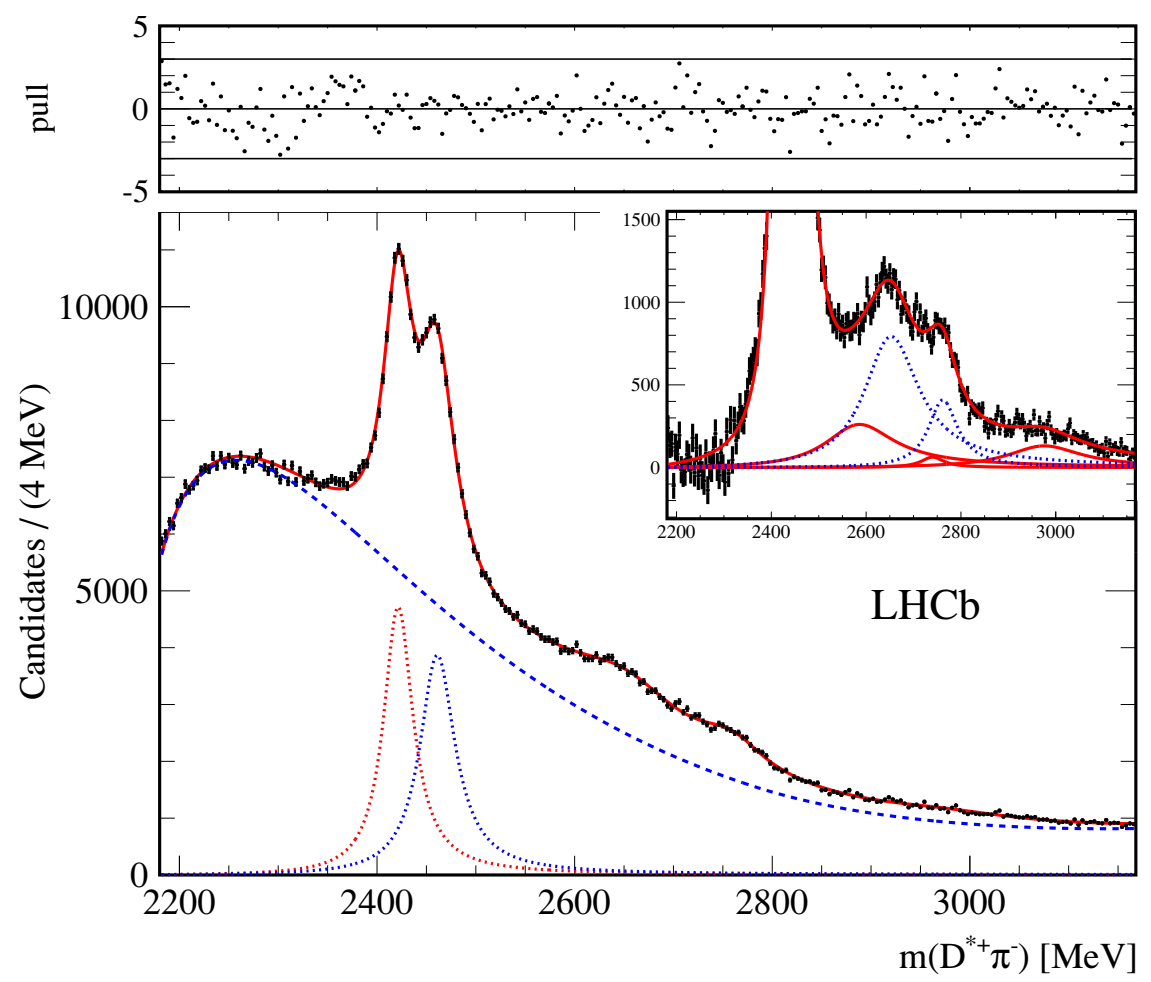

Figure 5. Fit to the natural parity sample $D^{*+} \pi^{-}$mass spectrum. The dashed (blue) line shows the fitted background, the dotted lines the $D_{1}(2420)^{0}$ (red) and $D_{2}^{*}(2460)^{0}$ (blue) contributions. The inset displays the $D^{*+} \pi^{-}$mass spectrum after subtracting the fitted background. The full line curves (red) show the contributions from $D_{J}(2580)^{0}, D_{J}(2740)^{0}$, and $D_{J}(3000)^{0}$. The dotted (blue) lines display the $D_{J}^{*}(2650)^{0}$ and $D_{J}^{*}(2760)^{0}$ contributions. The top window shows the pull distribution where the horizontal lines indicate $\pm 3 \sigma$.

hypothesis is also considered for $D_{J}(2580)^{0}$. The results from the fits are given in table 5 . In all cases unnatural parity is preferred over a natural parity assignment.

\section{$9 \quad$ Fit to the $D^{+} \pi^{-}$and $D^{0} \pi^{+}$mass spectra}

The $D^{+} \pi^{-}$and $D^{0} \pi^{+}$mass spectra consist of natural parity resonances. However these final states are affected by cross-feed from all the resonances that decay to the $D^{*} \pi$ final state. Figures 3(a) and (b) show (in the mass region around $2300 \mathrm{MeV}$ ) cross-feed contributions from $D_{1}(2420)$ and $D_{2}^{*}(2460)$ decays. However we also expect (in the mass region between 2400 and $2600 \mathrm{MeV}$ ) the presence of structures originating from the complex resonance structure present in the $D^{*} \pi$ mass spectrum in the mass region between 2500 and $2800 \mathrm{MeV}$.

To obtain an estimate of the lineshape and size of the cross-feed, we normalize the $D^{*+} \pi^{-}$mass spectrum to the $D^{+} \pi^{-}$mass spectrum using the sum of the $D_{1}(2420)^{0}$ and $D_{2}^{*}(2460)^{0}$ yields in the $D^{*+} \pi^{-}$mass spectrum $\left(N_{\text {sig }}\right)$ and the sum of the cross-feed in the $D^{+} \pi^{-}$mass spectrum $\left(N_{D^{+} \pi^{-}}^{\text {feed }}\right)$. We estimate that each resonance appearing in the $D^{*+} \pi^{-}$ should also appear in the $D^{+} \pi^{-}$mass spectrum with a yield given by

$$
N\left(D^{+} \pi^{-}\right)=N\left(D^{*+} \pi^{-}\right) R_{D^{+} \pi^{-}},
$$




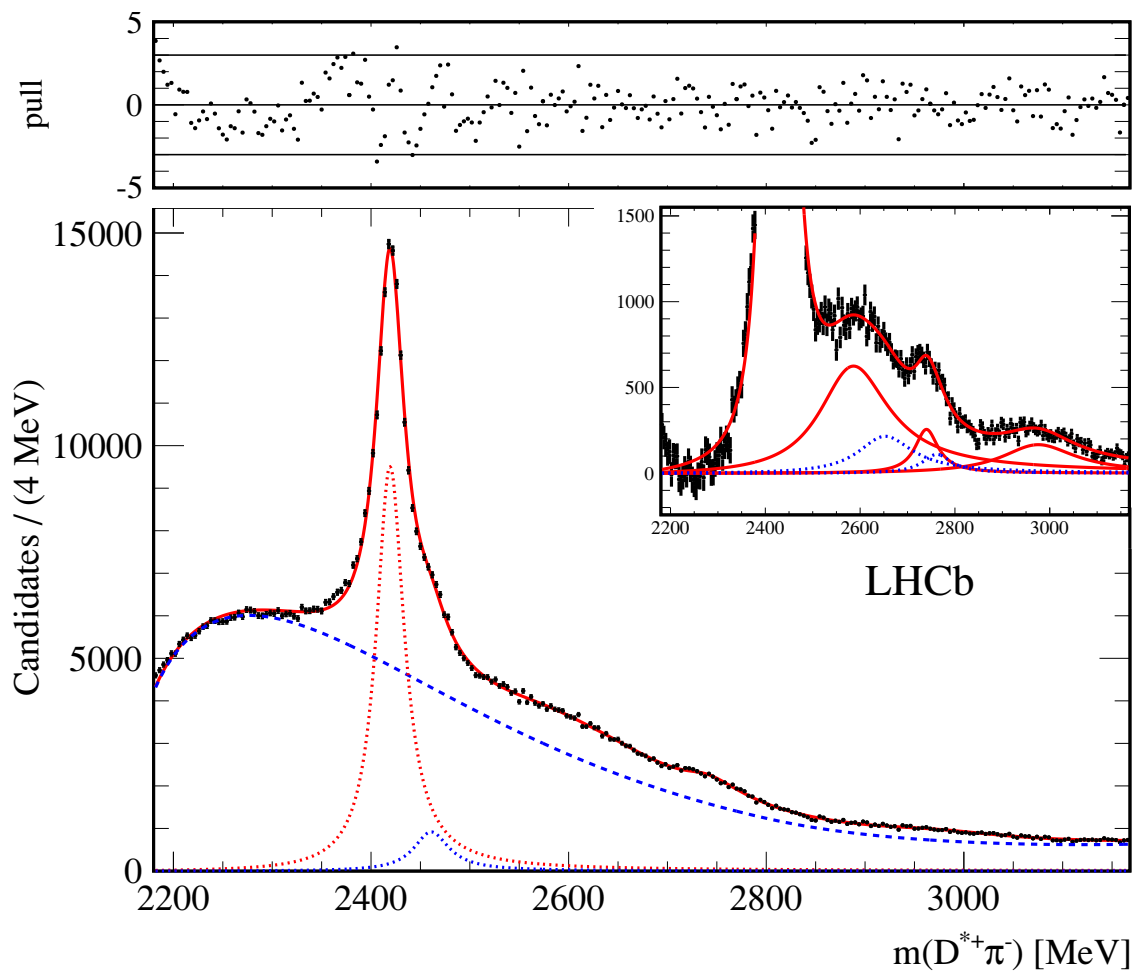

Figure 6. Fit to the unnatural parity sample $D^{*+} \pi^{-}$mass spectrum. The dashed (blue) line shows the fitted background, the dotted lines the $D_{1}(2420)^{0}$ (red) and $D_{2}^{*}(2460)^{0}$ (blue) contributions. The inset displays the $D^{*+} \pi^{-}$mass spectrum after subtracting the fitted background. The full line curves (red) show the contributions from $D_{J}(2580)^{0}, D_{J}(2740)^{0}$, and $D_{J}(3000)^{0}$ states. The dotted (blue) lines display the $D_{J}^{*}(2650)^{0}$ and $D_{J}^{*}(2760)^{0}$ contributions. The top window shows the pull distribution where the horizontal lines indicate $\pm 3 \sigma$.

where $R_{D^{+} \pi^{-}}=N_{D^{+} \pi^{-}}^{\text {feed }} / N_{\text {sig }}$. Here $N\left(D^{*+} \pi^{-}\right)$is the yield measured in the $D^{*+} \pi^{-}$final state, $N\left(D^{+} \pi^{-}\right)$is the expected yield in the $D^{+} \pi^{-}$mass spectrum and $R_{D^{+} \pi^{-}}=1.41 \pm 0.02$ where the uncertainty is statistical only.

Assuming similar yields for the $D_{1}(2420)^{+}$and $D_{2}^{*}(2460)^{+}$resonances, we estimate for the $D^{0} \pi^{+}$channel,

$$
N\left(D^{0} \pi^{+}\right)=N\left(D^{*+} \pi^{-}\right) R_{D^{0} \pi^{+}}
$$

where $R_{D^{0} \pi^{+}}=N_{D^{0} \pi^{+}}^{\text {feed }} / N_{\text {sig }}=1.87 \pm 0.02$ is the corresponding value for the $D^{0} \pi^{+}$channel.

To obtain the expected lineshape of the cross-feed in the $D^{+} \pi^{-}$final state, we perform a study based on a generator level simulation. We generate $D_{J}^{*}(2650)^{0}, D_{J}^{*}(2760)^{0}$, $D_{J}(2580)^{0}$ and $D_{J}(2740)^{0}$ decays according to the chain described in eq. (4.1). Given the small branching fraction of the $D^{*+} \rightarrow D^{+} \gamma$ decay, $(1.6 \pm 0.4) \%$, we only generate the $D^{*+} \rightarrow D^{+} \pi^{0}$ decay. The parameters of the resonances are as reported in table 4 and the decays to $D^{*+} \pi^{-}$are uniform over phase space. We then compute the resulting $D^{+} \pi^{-}$ mass spectra and normalize each contribution to the measured yields. The overall resulting structures are then scaled by the factor $R_{D^{+} \pi^{-}}$and superimposed on the $D^{+} \pi^{-}$mass spectrum shown in figure 11. 


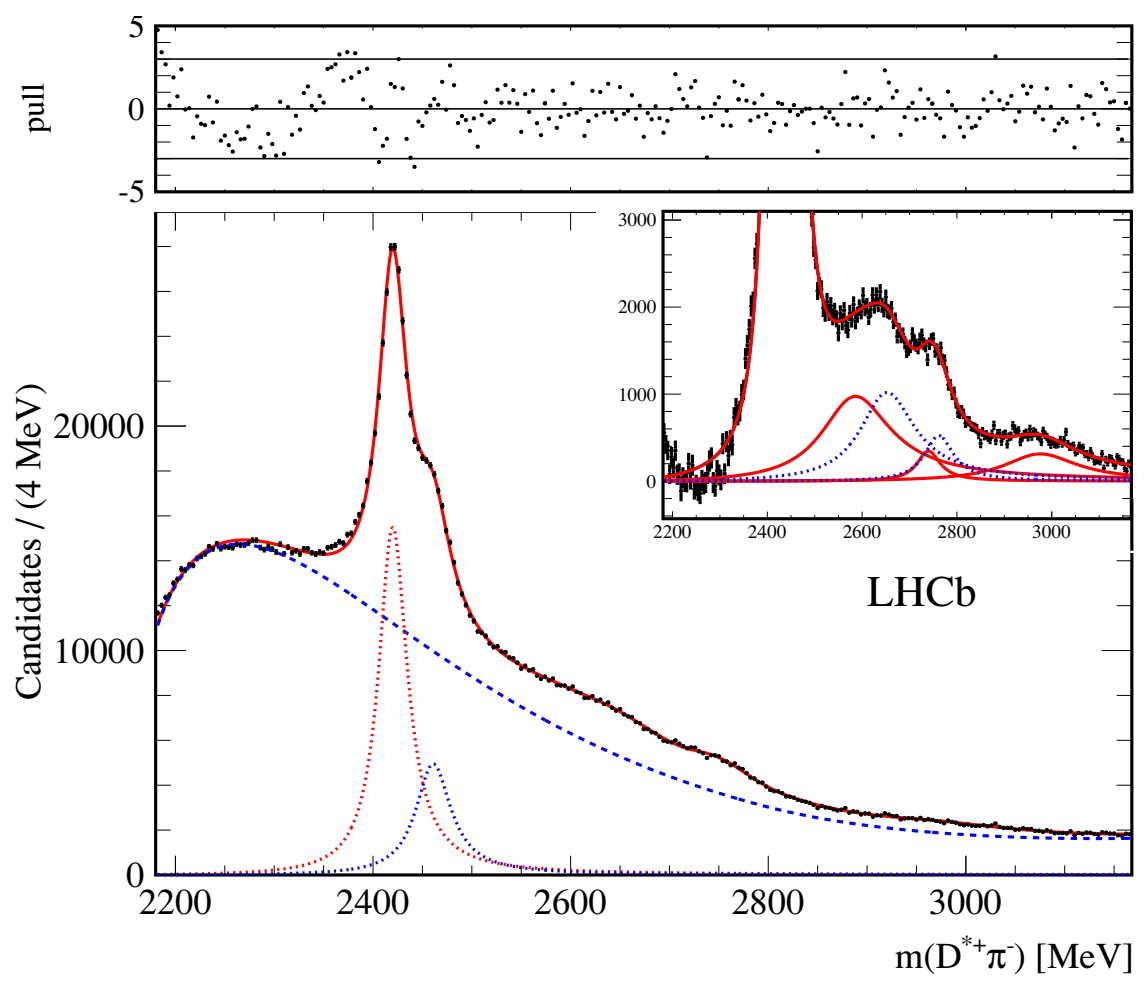

Figure 7. Fit to the total $D^{*+} \pi^{-}$sample. The dashed (blue) line shows the fitted background, the dotted lines the $D_{1}(2420)^{0}$ (red) and $D_{2}^{*}(2460)^{0}$ (blue) contributions. The inset displays the $D^{*+} \pi^{-}$mass spectrum after subtracting the fitted background. The full line curves (red) show the contributions from $D_{J}(2580)^{0}, D_{J}(2740)^{0}$, and $D_{J}(3000)^{0}$ states. The dotted (blue) lines display the $D_{J}^{*}(2650)^{0}$ and $D_{J}^{*}(2760)^{0}$ contributions. The top window shows the pull distribution where the horizontal lines indicate $\pm 3 \sigma$.
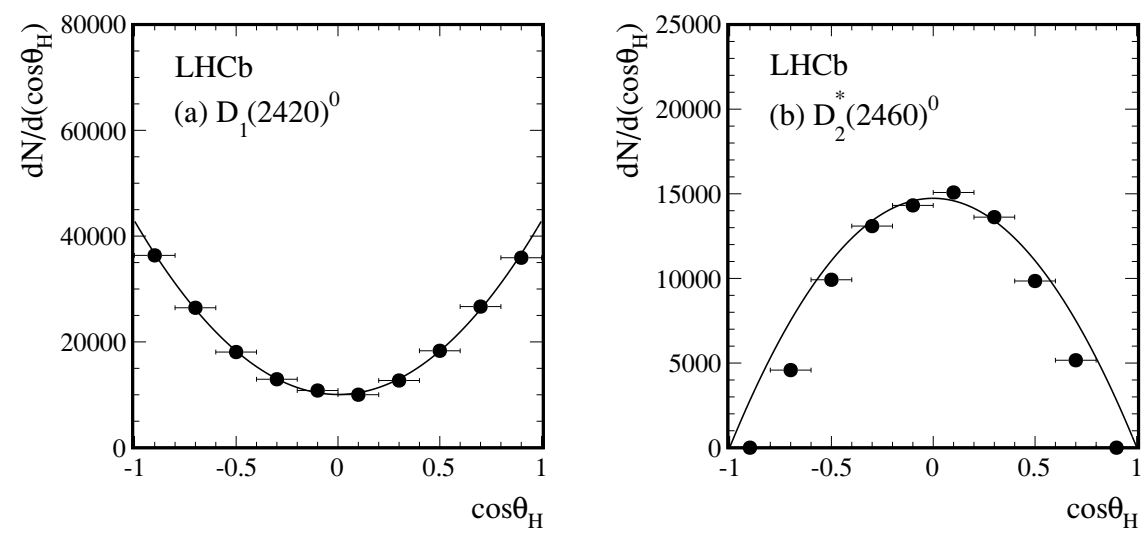

Figure 8. Distributions of (a) $D_{1}(2420)^{0}$ and (b) $D_{2}^{*}(2460)^{0}$ candidates as functions of the helicity angle $\cos \theta_{\mathrm{H}}$. The distributions are fitted with unnatural and natural parity functions, respectively.

Similarly, to obtain the expected lineshape of the cross-feed in the $D^{0} \pi^{+}$final state, we generate the four resonances according to the decays shown in eq. (4.2). We assume, for the charged modes, rates for the four states similar to that for the neutral modes. The 


\begin{tabular}{|c|c|c|c|c|c|}
\hline Resonance & $\begin{array}{l}\text { Final } \\
\text { state }\end{array}$ & Mass $(\mathrm{MeV})$ & Width $(\mathrm{MeV})$ & Yields $\times 10^{3}$ & $\begin{array}{c}\text { Significance } \\
(\sigma)\end{array}$ \\
\hline$D_{1}(2420)^{0}$ & $D^{*+} \pi^{-}$ & $2419.6 \pm \quad 0.1 \quad \pm 0.7$ & $35.2 \pm \quad 0.4 \quad \pm 0.9$ & $210.2 \pm 1.9 \pm 0.7$ & \\
\hline$D_{2}^{*}(2460)^{0}$ & $D^{*+} \pi^{-}$ & $2460.4 \pm \quad 0.4 \quad \pm 1.2$ & $43.2 \pm \quad 1.2 \pm 3.0$ & $81.9 \pm 1.2 \pm 0.9$ & \\
\hline$D_{J}^{*}(2650)^{0}$ & $D^{*+} \pi^{-}$ & $2649.2 \pm 3.5 \quad \pm 3.5$ & $140.2 \pm 17.1 \pm 18.6$ & $50.7 \pm 2.2 \pm 2.3$ & 24.5 \\
\hline$D_{J}^{*}(2760)^{0}$ & $D^{*+} \pi^{-}$ & $2761.1 \pm 5.1 \pm 6.5$ & $74.4 \pm 3.4 \pm 37.0$ & $14.4 \pm 1.7 \pm 1.7$ & 10.2 \\
\hline$D_{J}(2580)^{0}$ & $D^{*+} \pi^{-}$ & $2579.5 \pm 3.4 \pm 5.5$ & $177.5 \pm 17.8 \pm 46.0$ & $60.3 \pm 3.1 \pm 3.4$ & 18.8 \\
\hline$D_{J}(2740)^{0}$ & $D^{*+} \pi^{-}$ & $2737.0 \pm 3.5 \quad \pm 11.2$ & $73.2 \pm 13.4 \pm 25.0$ & $7.7 \pm 1.1 \pm 1.2$ & 7.2 \\
\hline$D_{J}(3000)^{0}$ & $D^{*+} \pi^{-}$ & $2971.8 \pm 8.7$ & $188.1 \pm 44.8$ & $9.5 \pm 1.1$ & 9.0 \\
\hline$D_{2}^{*}(2460)^{0}$ & $D^{+} \pi^{-}$ & $2460.4 \pm 0.1 \quad \pm 0.1$ & $45.6 \pm \quad 0.4 \pm 1.1$ & $675.0 \pm 9.0 \pm 1.3$ & \\
\hline$D_{J}^{*}(2760)^{0}$ & $D^{+} \pi^{-}$ & $2760.1 \pm 1.1 \pm 3.7$ & $74.4 \pm \quad 3.4 \quad \pm 19.1$ & $55.8 \pm 1.3 \pm 10.0$ & 17.3 \\
\hline$D_{J}^{*}(3000)^{0}$ & $D^{+} \pi^{-}$ & $3008.1 \pm 4.0$ & $110.5 \pm 11.5$ & $17.6 \pm 1.1$ & 21.2 \\
\hline$D_{2}^{*}(2460)^{+}$ & $D^{0} \pi^{+}$ & $2463.1 \pm \quad 0.2 \quad \pm 0.6$ & $48.6 \pm 1.3 \pm 1.9$ & $341.6 \pm 22.0 \pm 2.0$ & \\
\hline$D_{J}^{*}(2760)^{+}$ & $D^{0} \pi^{+}$ & $2771.7 \pm \quad 1.7 \pm 3.8$ & $66.7 \pm 6.6 \pm 10.5$ & $20.1 \pm 2.2 \pm 1.0$ & 18.8 \\
\hline$D_{J}^{*}(3000)^{+}$ & $D^{0} \pi^{+}$ & 3008.1 (fixed) & 110.5 (fixed) & $7.6 \pm 1.2$ & 6.6 \\
\hline
\end{tabular}

Table 4. Resonance parameters, yields and statistical significances. The first uncertainty is statistical, the second systematic. Significances are evaluated using the method described in the text.
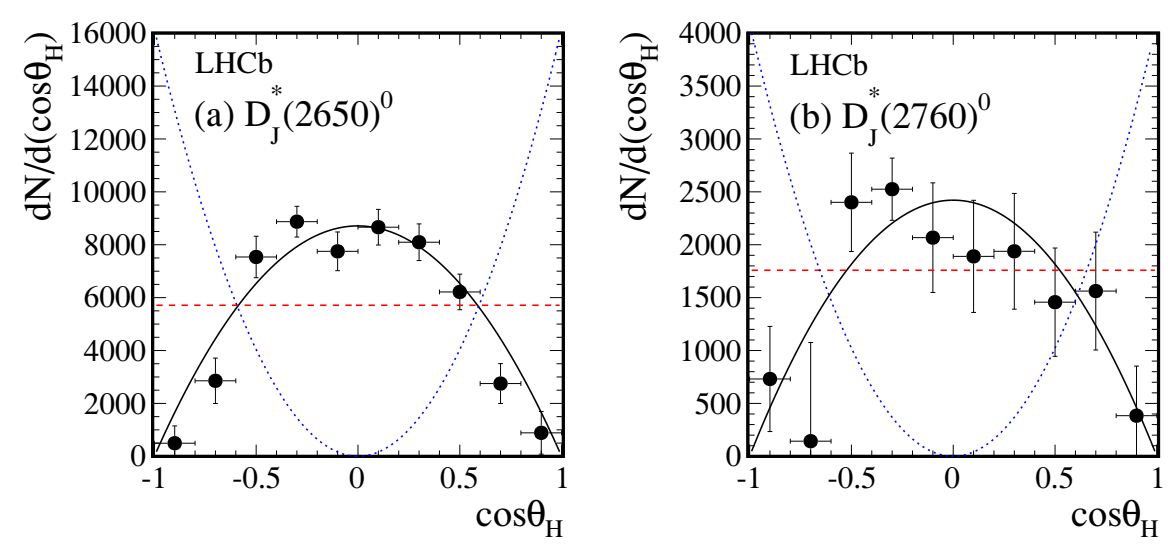

Figure 9. Distributions of (a) $D_{J}^{*}(2650)^{0}$ and (b) $D_{J}^{*}(2760)^{0}$ candidates as functions of the helicity angle $\cos \theta_{\mathrm{H}}$. The distributions are fitted with natural parity (black continuous), unnatural parity (red, dashed) and $J^{P}=0^{-}$(blue, dotted) functions.

overall resulting structures obtained for the $D^{* 0} \rightarrow D^{0} \pi^{0}$ and $D^{* 0} \rightarrow D^{0} \gamma$ decays are scaled according to their branching fractions and the distribution is scaled by the factor $R_{D^{0} \pi^{+}}$ discussed above. The resulting contribution is superimposed on the $D^{0} \pi^{+}$mass spectrum shown in figure 12 .

The cross-feed lineshapes obtained by the generator level simulation are not precise enough to be included in the fits to the $D^{+} \pi^{-}$and $D^{0} \pi^{+}$mass spectra. We therefore follow an empirical procedure to obtain good fits in this mass region. We first notice that these contributions produce a distortion of the $D_{2}^{*}(2460)^{0}$ and $D_{2}^{*}(2460)^{+}$lineshapes. 

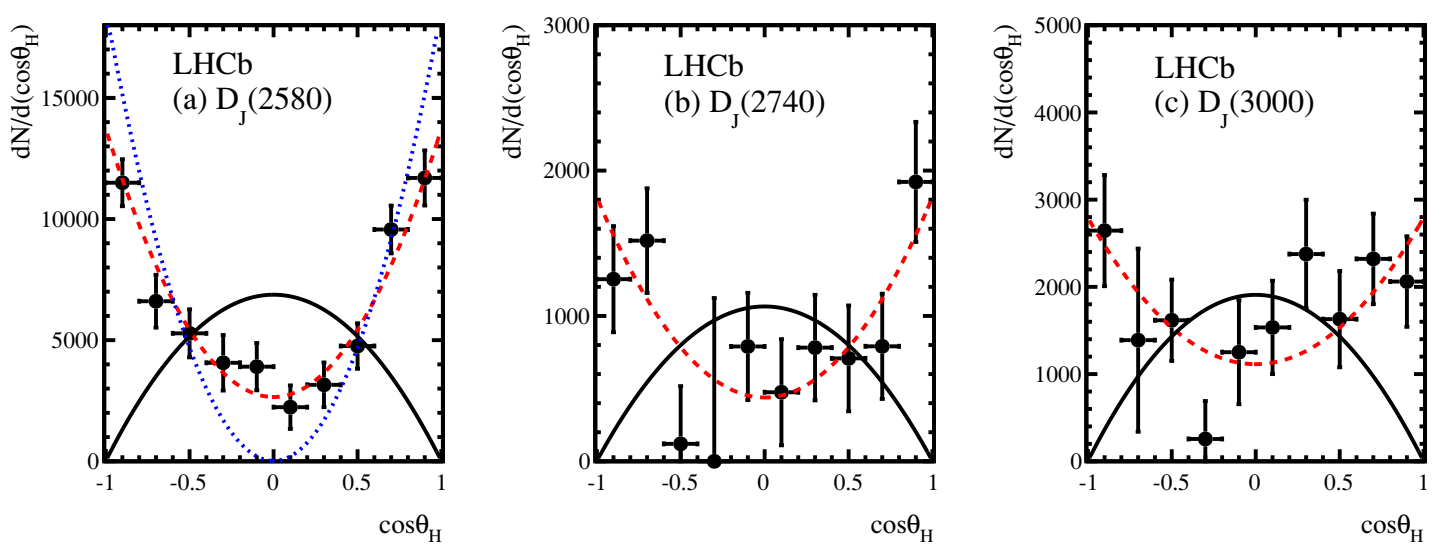

Figure 10. Distributions of (a) $D_{J}(2580)^{0}$, (b) $D_{J}(2740)^{0}$ and (c) $D_{J}(3000)^{0}$ candidates as functions of the helicity angle $\cos \theta_{\mathrm{H}}$. The distributions are fitted with natural parity (black continuous) and unnatural parity (red, dashed) functions. In (a) the $J^{P}=0^{-}$(blue, dotted) hypothesis is also tested.

\begin{tabular}{|c|c|c|c|c|c|c|c|}
\hline Resonance & $\begin{array}{c}J^{P} \\
\text { Function }\end{array}$ & $\chi^{2} / \mathrm{ndf}$ & $\begin{array}{c}J^{P} \\
\text { Function }\end{array}$ & $\chi^{2} / \mathrm{ndf}$ & $\begin{array}{c}J^{P} \\
\text { Function }\end{array}$ & $\chi^{2} / \mathrm{ndf}$ & $h$ Parameter \\
\hline$D_{1}(2420)^{0}$ & $\begin{array}{c}1^{+} \\
1+h \cos ^{2} \theta_{\mathrm{H}}\end{array}$ & $0.67 / 8$ & & & & & $3.30 \pm 0.48$ \\
\hline$D_{2}^{*}(2460)^{0}$ & $\begin{array}{c}2^{+} \\
\sin ^{2} \theta_{\mathrm{H}}\end{array}$ & $8.5 / 9$ & & & & & \\
\hline$D_{J}^{*}(2650)^{0}$ & $\begin{array}{l}\text { Natural } \\
\sin ^{2} \theta_{\mathrm{H}}\end{array}$ & $6.8 / 9$ & $\begin{array}{l}\text { unnatural } \\
\text { Const. }\end{array}$ & $200 / 9$ & $\begin{array}{c}0^{-} \\
\cos ^{2} \theta_{\mathrm{H}}\end{array}$ & $342 / 9$ & \\
\hline$D_{J}^{*}(2760)^{0}$ & $\begin{array}{c}\text { Natural } \\
\sin ^{2} \theta_{\mathrm{H}}\end{array}$ & $5.8 / 9$ & $\begin{array}{c}\text { unnatural } \\
\text { Const. }\end{array}$ & $26 / 9$ & $\begin{array}{c}0^{-} \\
\cos ^{2} \theta_{\mathrm{H}}\end{array}$ & $94 / 9$ & \\
\hline$D_{J}(2580)^{0}$ & $\begin{array}{l}\text { natural } \\
\sin ^{2} \theta_{\mathrm{H}}\end{array}$ & $151 / 9$ & $\begin{array}{r}\text { Unnatural } \\
1+h \cos ^{2} \theta_{\mathrm{H}}\end{array}$ & $3.4 / 8$ & $\begin{array}{c}0^{-} \\
\cos ^{2} \theta_{\mathrm{H}}\end{array}$ & $23 / 9$ & $4.2 \pm 1.3$ \\
\hline$D_{J}(2740)^{0}$ & $\begin{array}{c}\text { natural } \\
\sin ^{2} \theta_{\mathrm{H}}\end{array}$ & $34 / 9$ & $\begin{array}{r}\text { Unnatural } \\
1+h \cos ^{2} \theta_{\mathrm{H}}\end{array}$ & $6.6 / 8$ & & & $3.1 \pm 2.2$ \\
\hline$D_{J}(3000)^{0}$ & $\begin{array}{l}\text { natural } \\
\sin ^{2} \theta_{\mathrm{H}}\end{array}$ & $36.6 / 9$ & $\begin{array}{l}\text { Unnatural } \\
1+h \cos ^{2} \theta_{\mathrm{H}}\end{array}$ & $10 / 8$ & & & $1.5 \pm 0.9$ \\
\hline
\end{tabular}

Table 5. Results from the fits to the $\cos \theta_{\mathrm{H}}$ angular distributions for the resonances observed in the $D^{*+} \pi^{-}$mass spectrum. The resulting $\chi^{2} /$ ndf for different spin-parity assignments are reported. For each resonance, the expected angular distributions are indicated, where $h$ is a free parameter. The favoured spin-parity assignment is indicated in bold font.

These are accommodated in the fit by means of a Breit-Wigner function, which we include to obtain a good description of the data. The parameters of the Breit-Wigner function are $M=2414.3 \pm 1.4 \mathrm{MeV}$ and $\Gamma=103.2 \pm 2.7 \mathrm{MeV}$ for the $D^{+} \pi^{-}$final state and $M=$ $2435.1 \pm 5.2 \mathrm{MeV}$ and $\Gamma=106.9 \pm 6.2 \mathrm{MeV}$ for the $D^{0} \pi^{+}$final state. We consider these contributions as methods to improve the description of the cross-feeds. 


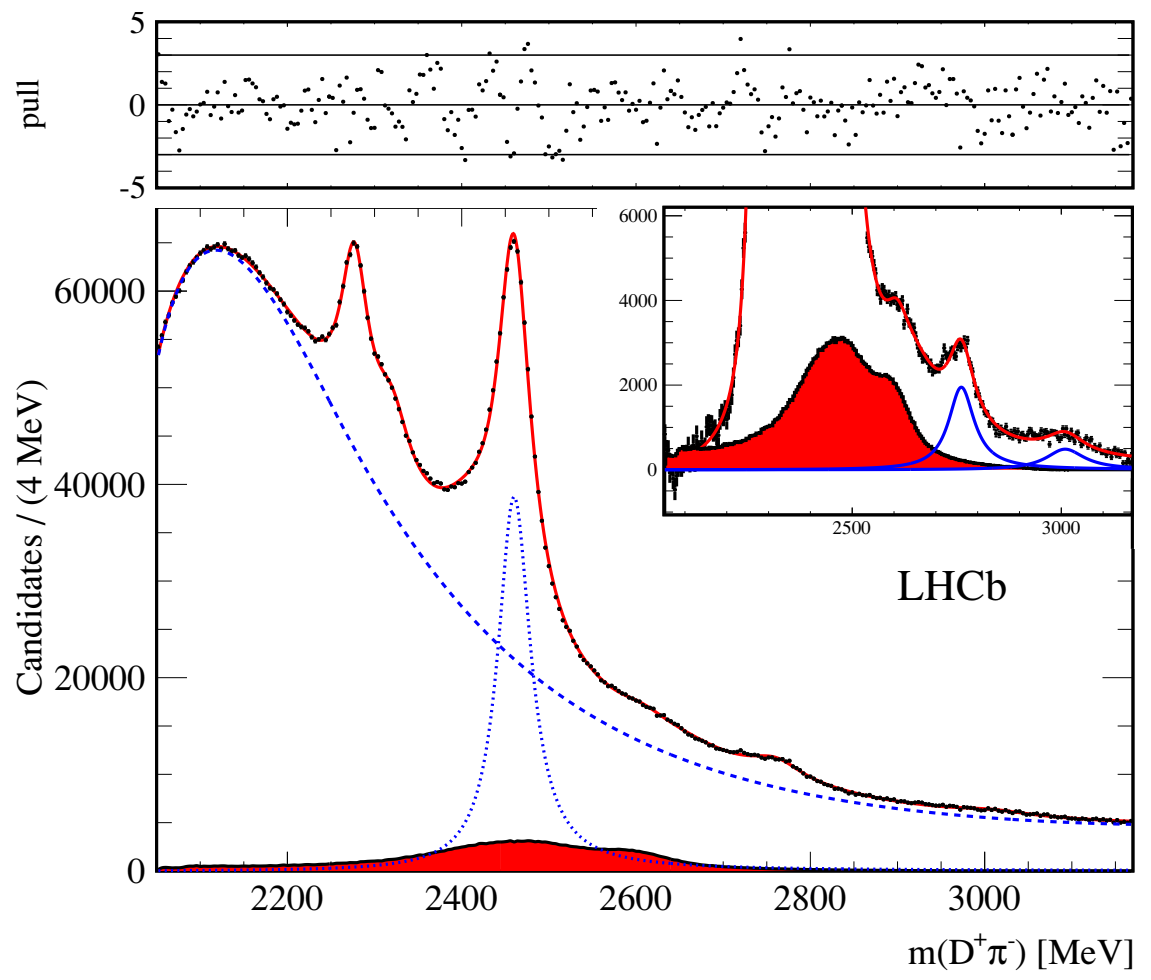

Figure 11. Fit to the $D^{+} \pi^{-}$mass spectrum. The filled histogram (in red) shows the estimated cross-feeds from the high mass $D^{*} \pi$ resonances. The dashed (blue) line displays the fitted background. The dotted (blue) line shows the $D_{2}^{*}(2460)^{0}$ contribution. The inset displays the mass spectrum after the fitted background subtraction. The full (blue) curves show the $D_{J}^{*}(2760)^{0}$ and $D_{J}^{*}(3000)^{0}$ contributions. The top window displays the pull distribution where the horizontal lines indicate $\pm 3 \sigma$.

We expect, in both the $D^{+} \pi^{-}$and $D^{0} \pi^{+}$mass spectra, the presence of $D_{J}^{*}(2650)$ and $D_{J}^{*}(2760)$ states. Enhancements in these mass regions can be seen in the two mass spectra shown in figure 11 and figure 12 . However the $D_{J}^{*}(2650)$ region is strongly affected by cross-feed. We include a simple Breit-Wigner function to describe these mass regions and obtain $M=2621.7 \pm 1.4 \mathrm{MeV}$ and $\Gamma=119.7 \pm 6.5 \mathrm{MeV}$ for the $D^{+} \pi^{-}$final state and $M=2599.9 \pm 0.9 \mathrm{MeV}$ and $\Gamma=72.3 \pm 4.0 \mathrm{MeV}$ for the $D^{0} \pi^{+}$final state. However the parameters so far obtained are strongly biased by the presence of the cross-feed and we therefore report, for the $D_{J}^{*}(2650)$ resonance, only the results obtained from the fit to the $D^{*+} \pi^{-}$mass spectrum.

To obtain good quality fits we add broad structures around $3000 \mathrm{MeV}$, which we label $D_{J}^{*}(3000)^{0}$ and $D_{J}^{*}(3000)^{+}$. Their parameters are derived from the fit to the $D^{+} \pi^{-}$mass spectrum and then fixed in the fit to the $D^{0} \pi^{+}$mass spectrum, where the effect is weaker.

The sensitivity of the fits to the presence of the broad $D_{0}^{*}(2400)$ resonance is tested by performing simulations that include a $D_{0}^{*}(2400)$ resonance with parameters fixed to their known values and vary the background lineshape within a wide range of values. We find a high correlation between the $D_{0}^{*}(2400)$ parameters and the background lineshape and a failure of the fit to obtain correct estimates of its parameters and yields. Therefore this contribution is not included in the fit. 


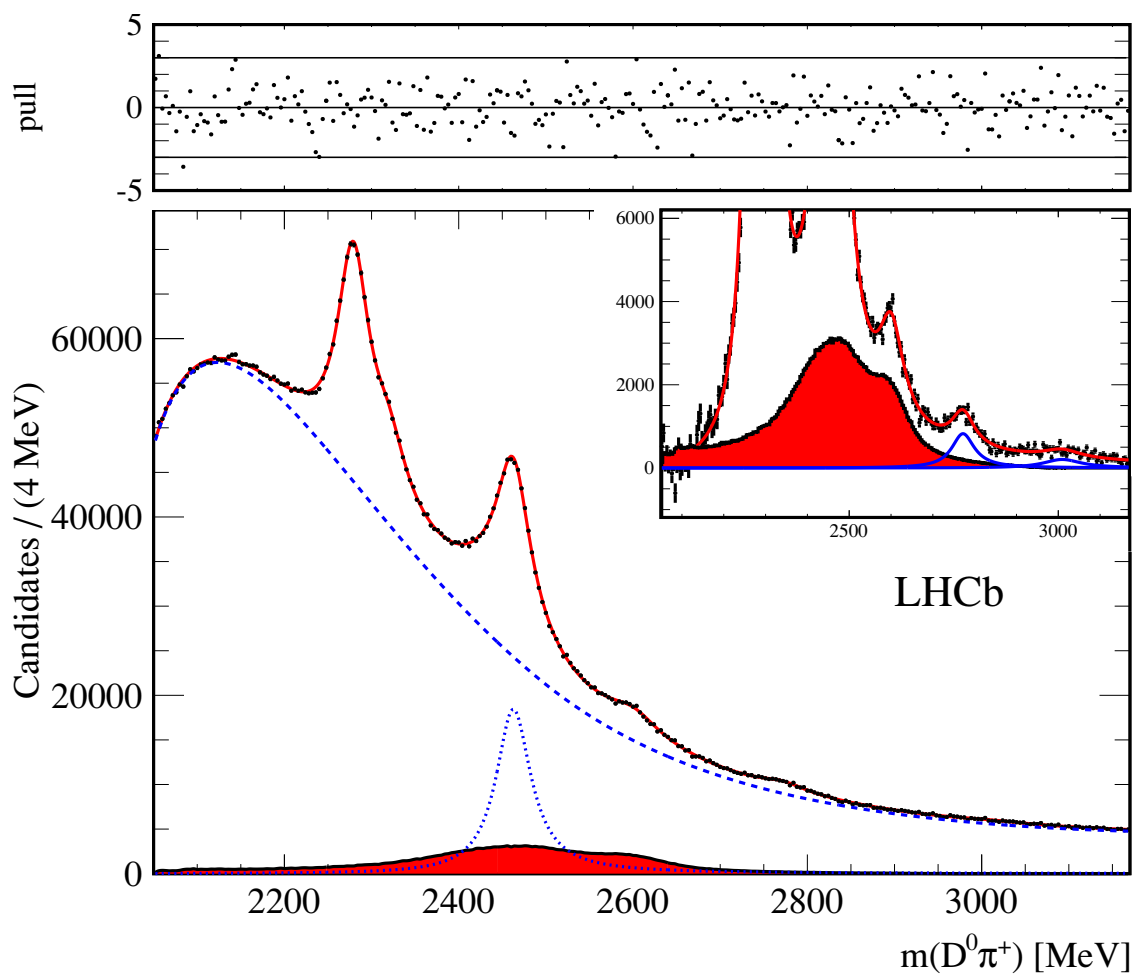

Figure 12. Fit to the $D^{0} \pi^{+}$mass spectrum. The filled histogram (in red) shows the estimated cross-feeds from the high mass $D^{*} \pi$ resonances. The dashed (blue) line displays the fitted background. The dotted (blue) line shows the $D_{2}^{*}(2460)^{+}$contribution. The inset displays the mass spectrum after the fitted background subtraction. The full (blue) curves show the $D_{J}^{*}(2760)^{+}$and $D_{J}^{*}(3000)^{+}$contributions. The top window displays the pull distribution where the horizontal lines indicate $\pm 3 \sigma$.

The fits to the $D^{+} \pi^{-}$and $D^{0} \pi^{+}$mass spectra are shown in figure 11 and figure 12 , respectively. Masses, widths, yields, and significances for the all the fitted resonances are displayed in table 4 . All the statistical significances are well above $5 \sigma$.

\section{Cross-checks and systematic uncertainties}

Several cross-checks are performed to test the stability of the fits and their correct statistical behaviour. We first repeat all the fits, including the spin-parity analysis, lowering the $p_{\mathrm{T}}$ requirement from 7.5 to $7.0 \mathrm{GeV}$. We find that all the resonance parameters vary within their statistical uncertainties and that the spin-parity assignments are not affected by this selection.

The fits stability and the uncertainties on the resonance parameters are tested using random variations of the histogram contents. For each histogram, we obtain and fit 500 new histograms by random Poisson variation of each bin content. We find in all cases a Gaussian behaviour of all the fit components with r.m.s. values that agree well with the statistical uncertainties given by the fits. 
The systematic uncertainties on the resonance parameters and yields reported in table 4 are estimated as follows. The background lineshape uncertainty is estimated using an alternative function $B(m)=\left(m-m_{\mathrm{th}}\right)^{a} e^{-b_{1} m-b_{2} m^{2}-b_{3} m^{3}}$, where $m_{\mathrm{th}}$ is the threshold mass. This function gives acceptable fits for the $D \pi$ mass spectra but generally a worse description of the threshold region.

The background lineshapes are additionaly tested by random variation of their parameters. For each mass spectrum, we generate and fit 500 new histograms where the resonance parameters and yields are fixed to the values obtained from the data, while the background yield is fixed but has parameters varying within $\pm 3 \sigma$ from the values obtained from the data. The distributions obtained from these fits are used to obtain systematic uncertainties due to the background lineshape. For the uncertainty due to the background lineshape the largest value between the estimates from the two methods described above is taken.

In the fits to the $D^{*+} \pi^{-}$mass spectra, where resonances have in some cases fixed parameters, we let the resonance parameters float sequentially. The procedure is repeated for each helicity sample and for the fit to the total mass spectrum. The Breit-Wigner shapes used to describe the $D_{J}^{*}(2760)$ resonance in the $D^{+} \pi^{-}$and $D^{0} \pi^{+}$mass spectra are replaced by a relativistic Breit-Wigner functions with different spin assignments. We also include the $D_{0}^{*}(2400)$ resonance with parameters fixed to the known values and obtain a small improvement in the fit to the $D^{+} \pi^{-}$mass spectrum but a yield consistent with zero in the fit to the $D^{0} \pi^{+}$mass spectrum.

The various estimated systematic uncertainties are added in quadrature. We do not report systematic uncertainties on the structures labelled as $D_{J}(3000)^{0}$ and $D_{J}^{*}(3000)^{+}$ because, being at the limit of the mass spectra, they are strongly correlated with the background parameters.

\section{Discussion and conclusions}

A study of the $D^{+} \pi^{-}, D^{0} \pi^{+}$, and $D^{*+} \pi^{-}$final states is reported using a sample of $p p$ collision data, corresponding to an integrated luminosity of $1.0 \mathrm{fb}^{-1}$, collected at a centreof-mass energy of $7 \mathrm{TeV}$ with the $\mathrm{LHCb}$ detector. We observe the $D_{1}(2420)^{0}$ resonance in the $D^{*+} \pi^{-}$final state, and the $D_{2}^{*}(2460)$ resonance in the $D^{+} \pi^{-}, D^{0} \pi^{+}$and $D^{*+} \pi^{-}$final states, measuring their parameters and confirming their spin-parity assignment [2]. We also observe two natural parity resonances $D_{J}^{*}(2650)^{0}$ and $D_{J}^{*}(2760)^{0}$ in the $D^{*+} \pi^{-}$mass spectrum and measure their angular distributions. The analysis of the $D^{+} \pi^{-}$and $D^{0} \pi^{+}$ mass spectra supports the presence of $D_{J}^{*}(2760)$ while the analysis of the $D_{J}^{*}(2650)$ region is inconclusive due to the presence of cross-feed from the resonances appearing in the $D^{*} \pi$ final state. The analysis of the $D^{*+} \pi^{-}$final state also shows the presence of two unnatural parity states, $D_{J}(2580)^{0}$ and $D_{J}(2740)^{0}$, for which we also perform a spin-parity analysis.

We observe a further structure in the $D^{*+} \pi^{-}$final state, labelled as $D_{J}(3000)^{0}$ with an angular distribution that is compatible with unnatural parity. We also observe structures in the $D^{+} \pi^{-}$and $D^{0} \pi^{+}$mass spectra that we label as $D_{J}^{*}(3000)^{0}$ and $D_{J}^{*}(3000)^{+}$. The properties of all these structures are uncertain and could be the result of a superposition of several $1 \mathrm{~F}$ states, as expected by the quark model predictions [1]. The overall results from 
this analysis are in partial agreement with the results from BaBar experiment [18], although for some resonances, especially the $D_{J}^{*}(2650)^{0}$ state, we measure different parameters.

The main source of the difference between the two analyses is related to the method of obtaining the $D_{J}^{*}(2650)^{0}$ parameters which, in the BaBar approach, are extracted from the fit to the $D^{+} \pi^{-}$mass spectrum and then fixed in the analysis of the $D^{*+} \pi^{-}$mass spectrum. Due to the correlation between the resonances parameters, this procedure also affects the properties of the other states appearing in the $D^{*+} \pi^{-}$mass spectrum. In the present analysis, as stated above, we measure important cross-feeds in the $2500-2600 \mathrm{MeV}$ region of the $D^{+} \pi^{-}$and $D^{0} \pi^{+}$final states and therefore we obtain the $D_{J}^{*}(2650)^{0}$ parameters from the $D^{*+} \pi^{-}$final state only.

We compare the quark-model predictions given in figure 1 with our mass measurements and spin-parity analysis reported in table 4 and table 5 , respectively. The BaBar analysis suggests a $J^{P}=0^{-}$assignment for the $D_{J}(2580)^{0}$ state (labelled $2 \mathrm{~S} D_{0}(2558)$ in figure 1). Our results are consistent with the BaBar measurement, but cannot confirm it, due to the superposition of many relatively broad resonances in a limited mass region which complicates the extraction of the resonances parameters.

The $D_{J}^{*}(2650)^{0}$ resonance is observed to decay to $D^{*+} \pi^{-}$, has natural parity and therefore is expected to decay to $D \pi$. However the presence of this state in the $D \pi$ mass spectra is obscured by the presence of cross-feeds from the $D^{*} \pi$ channels. We tentatively identify the $D_{J}^{*}(2650)^{0}$ resonance as a $J^{P}=1^{-}$state $\left(2 \mathrm{~S} D_{1}^{*}(2618)\right)$.

The $D_{J}^{*}(2760)^{0}$ is observed in the $D^{*+} \pi^{-}$and $D^{+} \pi^{-}$decay modes with consistent parameters. We also observe the $D_{J}^{*}(2760)^{+}$in the $D^{0} \pi^{+}$final state which can be identified as a $J^{P}=1^{-}$state $\left(1 \mathrm{D} D_{1}^{*}(2796)\right)$. The $D_{J}(2740)^{0}$ could be identified as the $J^{P}=2^{-}$ (1D $\left.D_{2}(2801)\right)$ resonance, although in this case the measured and predicted mass do not agree well. Definitive spin-parity assignments will be possible if these states are observed in $B$ decays.

\section{Acknowledgments}

We express our gratitude to our colleagues in the CERN accelerator departments for the excellent performance of the LHC. We thank the technical and administrative staff at the LHCb institutes. We acknowledge support from CERN and from the national agencies: CAPES, CNPq, FAPERJ and FINEP (Brazil); NSFC (China); CNRS/IN2P3 and Region Auvergne (France); BMBF, DFG, HGF and MPG (Germany); SFI (Ireland); INFN (Italy); FOM and NWO (The Netherlands); SCSR (Poland); MEN/IFA (Romania); MinES, Rosatom, RFBR and NRC "Kurchatov Institute" (Russia); MinECo, XuntaGal and GENCAT (Spain); SNSF and SER (Switzerland); NAS Ukraine (Ukraine); STFC (United Kingdom); NSF (U.S.A.). We also acknowledge the support received from the ERC under FP7. The Tier1 computing centres are supported by IN2P3 (France), KIT and BMBF (Germany), INFN (Italy), NWO and SURF (The Netherlands), PIC (Spain), GridPP (United Kingdom). We are thankful for the computing resources put at our disposal by Yandex LLC (Russia), as well as to the communities behind the multiple open source software packages that we depend on. 
Open Access. This article is distributed under the terms of the Creative Commons Attribution License which permits any use, distribution and reproduction in any medium, provided the original author(s) and source are credited.

\section{References}

[1] S. Godfrey and N. Isgur, Mesons in a relativized quark model with chromodynamics, Phys. Rev. D 32 (1985) 189 [INSPIRE].

[2] Particle Data Group collaboration, J. Beringer et al., Review of Particle Physics (RPP), Phys. Rev. D 86 (2012) 010001 [InSPIRE].

[3] Belle collaboration, K. Abe et al., Study of $B^{-} \rightarrow D^{* * 0} \pi^{-}\left(D^{* * 0} \rightarrow D^{(*)+} \pi^{-}\right)$decays, Phys. Rev. D 69 (2004) 112002 [hep-ex/0307021] [InSPIRE].

[4] BABAR collaboration, B. Aubert et al., Dalitz plot analysis of $B^{-} \rightarrow D^{+} \pi^{-} \pi^{-}$, Phys. Rev. D 79 (2009) 112004 [arXiv:0901.1291] [INSPIRE].

[5] P. Colangelo, F. De Fazio and R. Ferrandes, Bounding effective parameters in the chiral Lagrangian for excited heavy mesons, Phys. Lett. B 634 (2006) 235 [hep-ph/0511317] [INSPIRE].

[6] M. Di Pierro and E. Eichten, Excited heavy-light systems and hadronic transitions, Phys. Rev. D 64 (2001) 114004 [hep-ph/0104208] [INSPIRE].

[7] D. Ebert, R. Faustov and V. Galkin, Heavy-light meson spectroscopy and Regge trajectories in the relativistic quark model, Eur. Phys. J. C 66 (2010) 197 [arXiv:0910.5612] [INSPIRE].

[8] P. Colangelo, F. De Fazio, F. Giannuzzi and S. Nicotri, New meson spectroscopy with open charm and beauty, Phys. Rev. D 86 (2012) 054024 [arXiv:1207.6940] [InSPIRE].

[9] P. Colangelo and A. Khodjamirian, QCD sum rules, a modern perspective, hep-ph/0010175 [INSPIRE].

[10] T. Barnes, F. Close and H. Lipkin, Implications of a DK molecule at 2.32 GeV, Phys. Rev. D 68 (2003) 054006 [hep-ph/0305025] [INSPIRE].

[11] J. Vijande, F. Fernandez and A. Valcarce, Open-charm meson spectroscopy, Phys. Rev. D 73 (2006) 034002 [Erratum ibid. D 74 (2006) 059903] [hep-ph/0601143] [INSPIRE].

[12] G.S. Bali, The $D_{s J}(2317)$ : what can the lattice say?, Phys. Rev. D 68 (2003) 071501 [hep-ph/0305209] [INSPIRE].

[13] UKQCD collaboration, A. Dougall, R. Kenway, C. Maynard and C. McNeile, The spectrum of $D_{s}$ mesons from lattice QCD, Phys. Lett. B 569 (2003) 41 [hep-lat/0307001] [INSPIRE].

[14] H.-W. Lin, S. Ohta, A. Soni and N. Yamada, Charm as a domain wall fermion in quenched lattice QCD, Phys. Rev. D 74 (2006) 114506 [hep-lat/0607035] [INSPIRE].

[15] QCD collaboration, S. Dong et al., The Charmed-strange meson spectrum from overlap fermions on domain wall dynamical fermion configurations, PoS (LAT2009) 090 [arXiv:0911.0868] [INSPIRE].

[16] M. Gong et al., Study of the scalar charmed-strange meson $D_{s 0}^{*}(2317)$ with chiral fermions, PoS (LATTICE 2010) 106 [arXiv: 1103.0589] [INSPIRE].

[17] D. Mohler, S. Prelovsek and R.M. Woloshyn, D $\pi$ scattering and D meson resonances from lattice QCD, Phys. Rev. D 87 (2013) 034501 [arXiv:1208.4059] [InSPIRE]. 
[18] BaBAR collaboration, P. del Amo Sanchez et al., Observation of new resonances decaying to $D \pi$ and $D^{*} \pi$ in inclusive $e^{+} e^{-}$collisions near $\sqrt{s}=10.58$ GeV, Phys. Rev. D 82 (2010) 111101 [arXiv: 1009.2076] [INSPIRE].

[19] LHCB collaboration, The LHCb detector at the LHC, 2008 JINST 3 S08005 [INSPIRE].

[20] R. Aaij et al., The LHCb trigger and its performance in 2011, 2013 JINST 8 P04022 [arXiv: 1211.3055] [INSPIRE].

[21] M. Adinolfi et al., Performance of the LHCb RICH detector at the LHC, Eur. Phys. J. C 73 (2013) 2431 [arXiv: 1211.6759] [INSPIRE].

[22] T. Sjöstrand, S. Mrenna and P.Z. Skands, PYTHIA 6.4 physics and manual, JHEP 05 (2006) 026 [hep-ph/0603175] [INSPIRE].

[23] I. Belyaev et al., Handling of the generation of primary events in Gauss, the LHCb simulation framework, IEEE Nucl. Sci. Symp. Conf. Rec. 2010 (2010) 1155.

[24] D. Lange, The EvtGen particle decay simulation package, Nucl. Instrum. Meth. A 462 (2001) 152 [INSPIRE].

[25] GEANT4 collaboration, J. Allison et al., Geant4 developments and applications, IEEE Trans. Nucl. Sci. 53 (2006) 270.

[26] GEANT4 collaboration, S. Agostinelli et al., GEANT4: A Simulation toolkit, Nucl. Instrum. Meth. A 506 (2003) 250 [INSPIRE].

[27] LHCB collaboration, The LHCb simulation application, Gauss: Design, evolution and experience, J. Phys. Conf. Ser. 331 (2011) 032023 [inSPIRE].

[28] GENBOD, CERN Program Library.

[29] J.M. Blatt and V.F. Weisskopf, Theoretical nuclear physics, John Wiley \& Sons, New York U.S.A. (1952). 


\section{The LHCb collaboration}

R. Aaij ${ }^{40}$, B. Adeva ${ }^{36}$, M. Adinolfi ${ }^{45}$, C. Adrover ${ }^{6}$, A. Affolder ${ }^{51}$, Z. Ajaltouni ${ }^{5}$, J. Albrecht ${ }^{9}$, F. Alessio ${ }^{37}$, M. Alexander ${ }^{50}$, S. Ali ${ }^{40}$, G. Alkhazov ${ }^{29}$, P. Alvarez Cartelle ${ }^{36}$, A.A. Alves Jr ${ }^{24,37}$,

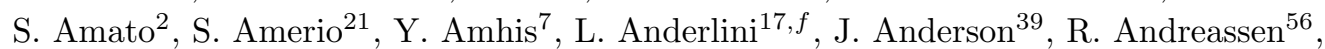
J.E. Andrews ${ }^{57}$, R.B. Appleby ${ }^{53}$, O. Aquines Gutierrez ${ }^{10}$, F. Archilli ${ }^{18}$, A. Artamonov ${ }^{34}$, M. Artuso ${ }^{58}$, E. Aslanides ${ }^{6}$, G. Auriemma ${ }^{24, m}$, M. Baalouch ${ }^{5}$, S. Bachmann ${ }^{11}$, J.J. Back ${ }^{47}$, C. Baesso ${ }^{59}$, V. Balagura ${ }^{30}$, W. Baldini ${ }^{16}$, R.J. Barlow ${ }^{53}$, C. Barschel ${ }^{37}$, S. Barsuk ${ }^{7}$, W. Barter ${ }^{46}$, Th. Bauer ${ }^{40}$, A. Bay ${ }^{38}$, J. Beddow ${ }^{50}$, F. Bedeschi ${ }^{22}$, I. Bediaga ${ }^{1}$, S. Belogurov ${ }^{30}$, K. Belous ${ }^{34}$, I. Belyaev ${ }^{30}$, E. Ben-Haim ${ }^{8}$, G. Bencivenni ${ }^{18}$, S. Benson ${ }^{49}$, J. Benton ${ }^{45}$, A. Berezhnoy ${ }^{31}$, R. Bernet ${ }^{39}$, M.-O. Bettler ${ }^{46}$, M. van Beuzekom ${ }^{40}$, A. Bien ${ }^{11}$, S. Bifani ${ }^{44}$, T. Bird ${ }^{53}$,

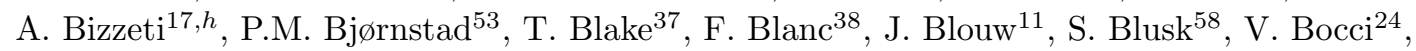
A. Bondar ${ }^{33}$, N. Bondar ${ }^{29}$, W. Bonivento ${ }^{15}$, S. Borghi ${ }^{53}$, A. Borgia ${ }^{58}$, T.J.V. Bowcock ${ }^{51}$, E. Bowen ${ }^{39}$, C. Bozzi ${ }^{16}$, T. Brambach ${ }^{9}$, J. van den Brand ${ }^{41}$, J. Bressieux ${ }^{38}$, D. Brett ${ }^{53}$,

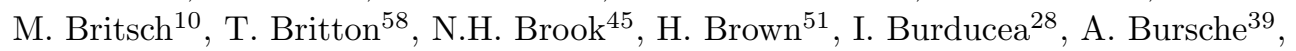
G. Busetto ${ }^{21, q}$, J. Buytaert ${ }^{37}$, S. Cadeddu ${ }^{15}$, O. Callot ${ }^{7}$, M. Calvi ${ }^{20, j}$, M. Calvo Gomez ${ }^{35, n}$, A. Camboni ${ }^{35}$, P. Campana ${ }^{18,37}$, D. Campora Perez ${ }^{37}$, A. Carbone ${ }^{14, c}$, G. Carboni ${ }^{23, k}$, R. Cardinale ${ }^{19, i}$, A. Cardini ${ }^{15}$, H. Carranza-Mejia ${ }^{49}$, L. Carson ${ }^{52}$, K. Carvalho Akiba ${ }^{2}$, G. Casse $^{51}$, L. Castillo Garcia ${ }^{37}$, M. Cattaneo ${ }^{37}$, Ch. Cauet ${ }^{9}$, R. Cenci ${ }^{57}$, M. Charles ${ }^{54}$, Ph. Charpentier ${ }^{37}$, P. Chen ${ }^{3,38}$, N. Chiapolini ${ }^{39}$, M. Chrzaszcz ${ }^{25}$, K. Ciba ${ }^{37}$, X. Cid Vidal ${ }^{37}$, G. Ciezarek ${ }^{52}$, P.E.L. Clarke ${ }^{49}$, M. Clemencic ${ }^{37}$, H.V. Cliff ${ }^{46}$, J. Closier ${ }^{37}$, C. Coca ${ }^{28}$, V. Coco ${ }^{40}$, J. Cogan ${ }^{6}$, E. Cogneras ${ }^{5}$, P. Collins ${ }^{37}$, A. Comerma-Montells ${ }^{35}$, A. Contu ${ }^{15,37}$, A. Cook ${ }^{45}$, M. Coombes ${ }^{45}$, S. Coquereau ${ }^{8}$, G. Corti ${ }^{37}$, B. Couturier ${ }^{37}$, G.A. Cowan ${ }^{49}$, D.C. Craik ${ }^{47}$, S. Cunliffe ${ }^{52}$, R. Currie ${ }^{49}$, C. D’Ambrosio ${ }^{37}$, P. David ${ }^{8}$, P.N.Y. David ${ }^{40}$, A. Davis ${ }^{56}$, I. De Bonis ${ }^{4}$, K. De Bruyn ${ }^{40}$, S. De Capua ${ }^{53}$, M. De Cian ${ }^{11}$, J.M. De Miranda ${ }^{1}$, L. De Paula ${ }^{2}$, W. De Silva ${ }^{56}$, P. De Simone ${ }^{18}$, D. Decamp ${ }^{4}$, M. Deckenhoff ${ }^{9}$, L. Del Buono ${ }^{8}$, N. Déléage ${ }^{4}$, D. Derkach ${ }^{54}$, O. Deschamps ${ }^{5}$, F. Dettori ${ }^{41}$, A. Di Canto ${ }^{11}$, H. Dijkstra ${ }^{37}$, M. Dogaru ${ }^{28}$, S. Donleavy ${ }^{51}$, F. Dordei ${ }^{11}$, A. Dosil Suárez ${ }^{36}$, D. Dossett ${ }^{47}$, A. Dovbnya ${ }^{42}$, F. Dupertuis ${ }^{38}$, P. Durante ${ }^{37}$, R. Dzhelyadin ${ }^{34}$, A. Dziurda ${ }^{25}$, A. Dzyuba ${ }^{29}$, S. Easo ${ }^{48,37}$, U. Egede ${ }^{52}$, V. Egorychev ${ }^{30}$, S. Eidelman ${ }^{33}$, D. van Eijk ${ }^{40}$, S. Eisenhardt ${ }^{49}$, U. Eitschberger ${ }^{9}$, R. Ekelhof ${ }^{9}$, L. Eklund ${ }^{50,37}$, I. El Rifai ${ }^{5}$, Ch. Elsasser ${ }^{39}$, A. Falabella ${ }^{14, e}$, C. Färber ${ }^{11}$, G. Fardell ${ }^{49}$, C. Farinelli ${ }^{40}$, S. Farry ${ }^{51}$, V. Fave ${ }^{38}$, D. Ferguson ${ }^{49}$, V. Fernandez Albor ${ }^{36}$, F. Ferreira Rodrigues ${ }^{1}$, M. Ferro-Luzzi ${ }^{37}$, S. Filippov ${ }^{32}$, M. Fiore ${ }^{16}$, C. Fitzpatrick ${ }^{37}$, M. Fontana ${ }^{10}$, F. Fontanelli ${ }^{19, i}$, R. Forty ${ }^{37}$,

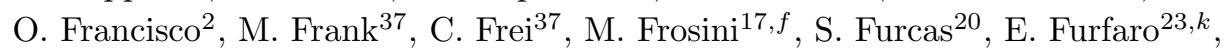

A. Gallas Torreira ${ }^{36}$, D. Galli ${ }^{14, c}$, M. Gandelman ${ }^{2}$, P. Gandini ${ }^{58}$, Y. Gao ${ }^{3}$, J. Garofoli ${ }^{58}$, P. Garosi ${ }^{53}$, J. Garra Tico ${ }^{46}$, L. Garrido ${ }^{35}$, C. Gaspar ${ }^{37}$, R. Gauld ${ }^{54}$, E. Gersabeck ${ }^{11}$, M. Gersabeck ${ }^{53}$, T. Gershon ${ }^{47,37}$, Ph. Ghez ${ }^{4}$, V. Gibson ${ }^{46}$, L. Giubega ${ }^{28}$, V.V. Gligorov ${ }^{37}$, C. Göbel ${ }^{59}$, D. Golubkov ${ }^{30}$, A. Golutvin ${ }^{52,30,37}$, A. Gomes ${ }^{2}$, H. Gordon ${ }^{54}$,

M. Grabalosa Gándara ${ }^{5}$, R. Graciani Diaz ${ }^{35}$, L.A. Granado Cardoso ${ }^{37}$, E. Graugés ${ }^{35}$, G. Graziani ${ }^{17}$, A. Grecu ${ }^{28}$, E. Greening ${ }^{54}$, S. Gregson ${ }^{46}$, P. Griffith ${ }^{44}$, O. Grünberg ${ }^{60}$, B. Gui ${ }^{58}$, E. Gushchin ${ }^{32}$, Yu. Guz ${ }^{34,37}$, T. Gys ${ }^{37}$, C. Hadjivasiliou ${ }^{58}$, G. Haefeli ${ }^{38}$, C. Haen ${ }^{37}$, S.C. Haines ${ }^{46}$, S. Hall ${ }^{52}$, B. Hamilton ${ }^{57}$, T. Hampson ${ }^{45}$, S. Hansmann-Menzemer ${ }^{11}$, N. Harnew ${ }^{54}$, S.T. Harnew ${ }^{45}$,

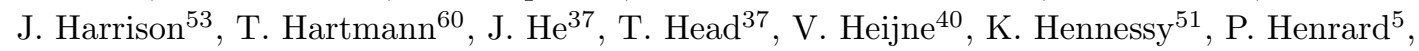
J.A. Hernando Morata ${ }^{36}$, E. van Herwijnen ${ }^{37}$, A. Hicheur ${ }^{1}$, E. Hicks ${ }^{51}$, D. Hill ${ }^{54}$, M. Hoballah ${ }^{5}$, C. Hombach ${ }^{53}$, P. Hopchev 4 , W. Hulsbergen ${ }^{40}$, P. Hunt ${ }^{54}$, T. Huse ${ }^{51}$, N. Hussain ${ }^{54}$, D. Hutchcroft ${ }^{51}$, D. Hynds ${ }^{50}$, V. Iakovenko ${ }^{43}$, M. Idzik ${ }^{26}$, P. Ilten ${ }^{12}$, R. Jacobsson ${ }^{37}$, A. Jaeger ${ }^{11}$, E. Jans ${ }^{40}$, P. Jaton ${ }^{38}$, A. Jawahery ${ }^{57}$, F. Jing ${ }^{3}$, M. John ${ }^{54}$, D. Johnson ${ }^{54}$, C.R. Jones ${ }^{46}$,

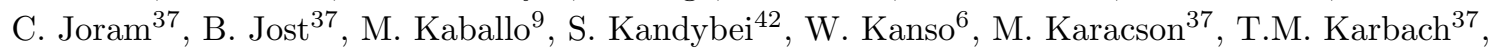


I.R. Kenyon ${ }^{44}$, T. Ketel ${ }^{41}$, A. Keune ${ }^{38}$, B. Khanji ${ }^{20}$, O. Kochebina ${ }^{7}$, I. Komarov ${ }^{38}$, R.F. Koopman ${ }^{41}$, P. Koppenburg ${ }^{40}$, M. Korolev ${ }^{31}$, A. Kozlinskiy ${ }^{40}$, L. Kravchuk ${ }^{32}$, K. Kreplin ${ }^{11}$, M. Kreps ${ }^{47}$, G. Krocker ${ }^{11}$, P. Krokovny ${ }^{33}$, F. Kruse ${ }^{9}$, M. Kucharczyk ${ }^{20,25, j}$, V. Kudryavtses ${ }^{33}$, T. Kvaratskheliya ${ }^{30,37}$, V.N. La Thi ${ }^{38}$, D. Lacarrere ${ }^{37}$, G. Lafferty ${ }^{53}$, A. Lai ${ }^{15}$, D. Lambert ${ }^{49}$, R.W. Lambert ${ }^{41}$, E. Lanciotti ${ }^{37}$, G. Lanfranchi ${ }^{18}$, C. Langenbruch ${ }^{37}$, T. Latham ${ }^{47}$, C. Lazzeroni ${ }^{44}$, R. Le Gac ${ }^{6}$, J. van Leerdam ${ }^{40}$, J.-P. Lees ${ }^{4}$, R. Lefèvre ${ }^{5}$, A. Leflat ${ }^{31}$, J. Lefrançois ${ }^{7}$,

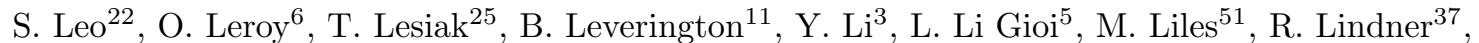

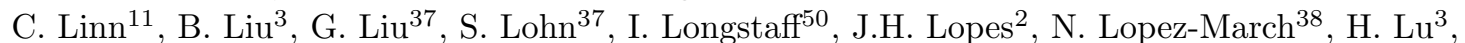
D. Lucchesi ${ }^{21, q}$, J. Luisier ${ }^{38}$, H. Luo ${ }^{49}$, F. Machefert ${ }^{7}$, I.V. Machikhiliyan ${ }^{4,30}$, F. Maciuc ${ }^{28}$, O. Maev ${ }^{29,37}$, S. Malde ${ }^{54}$, G. Manca ${ }^{15, d}$, G. Mancinelli ${ }^{6}$, J. Maratas ${ }^{5}$, U. Marconi ${ }^{14}$, R. Märki ${ }^{38}$, J. Marks ${ }^{11}$, G. Martellotti ${ }^{24}$, A. Martens ${ }^{8}$, A. Martín Sánchez ${ }^{7}$, M. Martinelli ${ }^{40}$, D. Martinez Santos ${ }^{41}$, D. Martins Tostes ${ }^{2}$, A. Massafferri ${ }^{1}$, R. Matev ${ }^{37}$, Z. Mathe ${ }^{37}$, C. Matteuzzi ${ }^{20}$, E. Maurice ${ }^{6}$, A. Mazurov ${ }^{16,32,37, e}$, B. Mc Skelly ${ }^{51}$, J. McCarthy ${ }^{44}$, A. McNab $^{53}$, R. McNulty ${ }^{12}$, B. Meadows ${ }^{56,54}$, F. Meier ${ }^{9}$, M. Meissner ${ }^{11}$, M. Merk ${ }^{40}$, D.A. Milanes ${ }^{8}$, M.-N. Minard ${ }^{4}$, J. Molina Rodriguez ${ }^{59}$, S. Monteil ${ }^{5}$, D. Moran ${ }^{53}$, P. Morawski ${ }^{25}$, A. Mordà ${ }^{6}$, M.J. Morello ${ }^{22, s}$, R. Mountain ${ }^{58}$, I. Mous ${ }^{40}$, F. Muheim ${ }^{49}$, K. Müller ${ }^{39}$, R. Muresan ${ }^{28}$, B. Muryn ${ }^{26}$, B. Muster ${ }^{38}$, P. Naik ${ }^{45}$, T. Nakada ${ }^{38}$, R. Nandakumar ${ }^{48}$, I. Nasteva ${ }^{1}$, M. Needham ${ }^{49}$, S. Neubert ${ }^{37}$, N. Neufeld ${ }^{37}$, A.D. Nguyen ${ }^{38}$, T.D. Nguyen ${ }^{38}$, C. Nguyen-Mau ${ }^{38, o}$, M. Nicol ${ }^{7}$, V. Niess ${ }^{5}$, R. Niet ${ }^{9}$, N. Nikitin ${ }^{31}$, T. Nikodem ${ }^{11}$, A. Nomerotski ${ }^{54}$, A. Novoselov ${ }^{34}$,

A. Oblakowska-Mucha ${ }^{26}$, V. Obraztsov ${ }^{34}$, S. Oggero ${ }^{40}$, S. Ogilvy ${ }^{50}$, O. Okhrimenko ${ }^{43}$, R. Oldeman ${ }^{15, d}$, M. Orlandea ${ }^{28}$, J.M. Otalora Goicochea ${ }^{2}$, P. Owen ${ }^{52}$, A. Oyanguren ${ }^{35}$, B.K. Pal ${ }^{58}$, A. Palano ${ }^{13, b}$, M. Palutan ${ }^{18}$, J. Panman $^{37}$, A. Papanestis ${ }^{48}$, M. Pappagallo ${ }^{50}$, C. Parkes $^{53}$, C.J. Parkinson ${ }^{52}$, G. Passaleva ${ }^{17}$, G.D. Patel ${ }^{51}$, M. Patel ${ }^{52}$, G.N. Patrick ${ }^{48}$, C. Patrignani ${ }^{19, i}$, C. Pavel-Nicorescu ${ }^{28}$, A. Pazos Alvarez ${ }^{36}$, A. Pellegrino ${ }^{40}$, G. Penso ${ }^{24, l}$, M. Pepe Altarelli ${ }^{37}$, S. Perazzini ${ }^{14, c}$, E. Perez Trigo ${ }^{36}$, A. Pérez-Calero Yzquierdo ${ }^{35}$, P. Perret ${ }^{5}$, M. Perrin-Terrin ${ }^{6}$, G. Pessina ${ }^{20}$, K. Petridis ${ }^{52}$, A. Petrolini ${ }^{19, i}$, A. Phan ${ }^{58}$, E. Picatoste Olloqui ${ }^{35}$, B. Pietrzyk ${ }^{4}$, T. Pilař ${ }^{47}$, D. Pinci ${ }^{24}$, S. Playfer ${ }^{49}$, M. Plo Casasus ${ }^{36}$, F. Polci ${ }^{8}$, G. Polok ${ }^{25}$, A. Poluektov ${ }^{47,33}$, E. Polycarpo ${ }^{2}$, A. Popov ${ }^{34}$, D. Popov ${ }^{10}$, B. Popovici ${ }^{28}$, C. Potterat ${ }^{35}$, A. Powell ${ }^{54}$, J. Prisciandaro ${ }^{38}$, A. Pritchard ${ }^{51}$, C. Prouve ${ }^{7}$, V. Pugatch ${ }^{43}$, A. Puig Navarro ${ }^{38}$, G. Punzi ${ }^{22, r}$, W. Qian ${ }^{4}$, J.H. Rademacker ${ }^{45}$, B. Rakotomiaramanana ${ }^{38}$, M.S. Rangel ${ }^{2}$, I. Raniuk ${ }^{42}$, N. Rauschmayr ${ }^{37}$, G. Raven ${ }^{41}$, S. Redford ${ }^{54}$, M.M. Reid ${ }^{47}$, A.C. dos Reis ${ }^{1}$, S. Ricciardi ${ }^{48}$, A. Richards ${ }^{52}$, K. Rinnert ${ }^{51}$, V. Rives Molina ${ }^{35}$, D.A. Roa Romero ${ }^{5}$, P. Robbe ${ }^{7}$, D.A. Roberts ${ }^{57}$, E. Rodrigues ${ }^{53}$, P. Rodriguez Perez ${ }^{36}$, S. Roiser ${ }^{37}$, V. Romanovsky ${ }^{34}$, A. Romero Vidal ${ }^{36}$, J. Rouvinet ${ }^{38}$, T. Ruf ${ }^{37}$, F. Ruffini ${ }^{22}$, H. Ruiz ${ }^{35}$, P. Ruiz Valls ${ }^{35}$, G. Sabatino ${ }^{24, k}$, J.J. Saborido Silva ${ }^{36}$, N. Sagidova ${ }^{29}$, P. Sail ${ }^{50}$, B. Saitta ${ }^{15, d}$, V. Salustino Guimaraes ${ }^{2}$, C. Salzmann ${ }^{39}$, B. Sanmartin Sedes ${ }^{36}$, M. Sannino ${ }^{19, i}$, R. Santacesaria ${ }^{24}$, C. Santamarina Rios ${ }^{36}$, E. Santovetti ${ }^{23, k}$, M. Sapunov ${ }^{6}$, A. Sarti ${ }^{18, l}$, C. Satriano ${ }^{24, m}$, A. Satta ${ }^{23}$, M. Savrie ${ }^{16, e}$, D. Savrina ${ }^{30,31}$, P. Schaack ${ }^{52}$, M. Schiller ${ }^{41}$, H. Schindler ${ }^{37}$, M. Schlupp ${ }^{9}$, M. Schmelling ${ }^{10}$, B. Schmidt ${ }^{37}$, O. Schneider ${ }^{38}$, A. Schopper ${ }^{37}$, M.-H. Schune ${ }^{7}$, R. Schwemmer ${ }^{37}$, B. Sciascia ${ }^{18}$, A. Sciubba ${ }^{24}$, M. Seco ${ }^{36}$, A. Semennikov ${ }^{30}$, K. Senderowska ${ }^{26}$, I. Sepp ${ }^{52}$, N. Serra ${ }^{39}$, J. Serrano ${ }^{6}$, P. Seyfert ${ }^{11}$, M. Shapkin ${ }^{34}$, I. Shapoval ${ }^{16,42}$, P. Shatalov ${ }^{30}$, Y. Shcheglov ${ }^{29}$, T. Shears ${ }^{51,37}$, L. Shekhtman ${ }^{33}$, O. Shevchenko ${ }^{42}$, V. Shevchenko ${ }^{30}$, A. Shires ${ }^{52}$, R. Silva Coutinho ${ }^{47}$, M. Sirendi ${ }^{46}$, T. Skwarnicki ${ }^{58}$, N.A. Smith ${ }^{51}$, E. Smith ${ }^{54,48}$, J. Smith ${ }^{46}$, M. Smith ${ }^{53}$, M.D. Sokoloff ${ }^{56}$, F.J.P. Soler ${ }^{50}$, F. Soomro ${ }^{18}$, D. Souza ${ }^{45}$, B. Souza De Paula ${ }^{2}$, B. Spaan ${ }^{9}$, A. Sparkes ${ }^{49}$, P. Spradlin ${ }^{50}$, F. Stagni ${ }^{37}$, S. Stahl ${ }^{11}$, O. Steinkamp ${ }^{39}$, S. Stevenson ${ }^{54}$, S. Stoica ${ }^{28}$, S. Stone ${ }^{58}$, B. Storaci ${ }^{39}$, M. Straticiuc ${ }^{28}$, U. Straumann ${ }^{39}$, V.K. Subbiah ${ }^{37}$, L. Sun ${ }^{56}$, S. Swientek ${ }^{9}$, V. Syropoulos ${ }^{41}$, M. Szczekowski ${ }^{27}$, P. Szczypka ${ }^{38,37}$, T. Szumlak ${ }^{26}$, S. T'Jampens ${ }^{4}$,

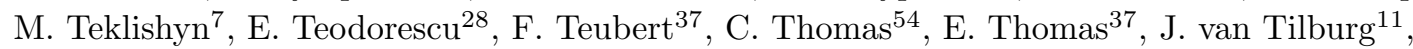




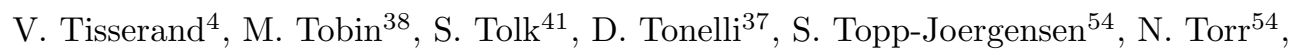

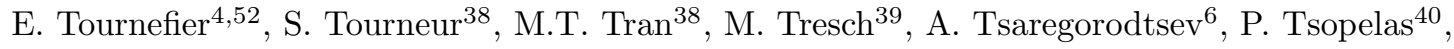
N. Tuning ${ }^{40}$, M. Ubeda Garcia ${ }^{37}$, A. Ukleja ${ }^{27}$, D. Urner ${ }^{53}$, A. Ustyuzhanin ${ }^{52, p}$, U. Uwer ${ }^{11}$, V. Vagnoni ${ }^{14}$, G. Valenti ${ }^{14}$, A. Vallier ${ }^{7}$, M. Van Dijk ${ }^{45}$, R. Vazquez Gomez ${ }^{18}$, P. Vazquez Regueiro ${ }^{36}$, C. Vázquez Sierra ${ }^{36}$, S. Vecchi ${ }^{16}$, J.J. Velthuis ${ }^{45}$, M. Veltri ${ }^{17, g}$, G. Veneziano ${ }^{38}$, M. Vesterinen ${ }^{37}$, B. Viaud ${ }^{7}$, D. Vieira ${ }^{2}$, X. Vilasis-Cardona ${ }^{35, n}$, A. Vollhardt ${ }^{39}$, D. Volyanskyy ${ }^{10}$, D. Voong ${ }^{45}$, A. Vorobyev ${ }^{29}$, V. Vorobyev ${ }^{33}$, C. Voß ${ }^{60}$, H. Voss ${ }^{10}$, R. Waldi ${ }^{60}$, C. Wallace ${ }^{47}$, R. Wallace ${ }^{12}$, S. Wandernoth ${ }^{11}$, J. Wang ${ }^{58}$, D.R. Ward ${ }^{46}$, N.K. Watson ${ }^{44}$,

A.D. Webber ${ }^{53}$, D. Websdale ${ }^{52}$, M. Whitehead ${ }^{47}$, J. Wicht ${ }^{37}$, J. Wiechczynski ${ }^{25}$, D. Wiedner ${ }^{11}$,

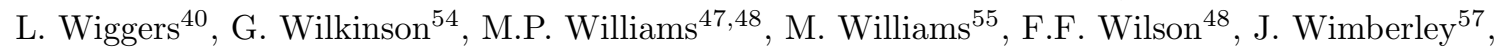
J. Wishahi ${ }^{9}$, M. Witek ${ }^{25}$, S.A. Wotton ${ }^{46}$, S. Wright ${ }^{46}$, S. Wu ${ }^{3}$, K. Wyllie ${ }^{37}$, Y. Xie ${ }^{49,37}$, Z. Xing ${ }^{58}$, Z. Yang ${ }^{3}$, R. Young ${ }^{4}$, X. Yuan ${ }^{3}$, O. Yushchenko ${ }^{34}$, M. Zangoli ${ }^{14}$, M. Zavertyaev ${ }^{10, a}$, F. Zhang ${ }^{3}$, L. Zhang ${ }^{58}$, W.C. Zhang ${ }^{12}$, Y. Zhang ${ }^{3}$, A. Zhelezov ${ }^{11}$, A. Zhokhov ${ }^{30}$, L. Zhong ${ }^{3}$ and A. Zvyagin ${ }^{37}$

1 Centro Brasileiro de Pesquisas Físicas (CBPF), Rio de Janeiro, Brazil

2 Universidade Federal do Rio de Janeiro (UFRJ), Rio de Janeiro, Brazil

3 Center for High Energy Physics, Tsinghua University, Beijing, China

4 LAPP, Université de Savoie, CNRS/IN2P3, Annecy-Le-Vieux, France

5 Clermont Université, Université Blaise Pascal, CNRS/IN2P3, LPC, Clermont-Ferrand, France

6 CPPM, Aix-Marseille Université, CNRS/IN2P3, Marseille, France

7 LAL, Université Paris-Sud, CNRS/IN2P3, Orsay, France

8 LPNHE, Université Pierre et Marie Curie, Université Paris Diderot, CNRS/IN2P3, Paris, France

9 Fakultät Physik, Technische Universität Dortmund, Dortmund, Germany

10 Max-Planck-Institut für Kernphysik (MPIK), Heidelberg, Germany

11 Physikalisches Institut, Ruprecht-Karls-Universität Heidelberg, Heidelberg, Germany

12 School of Physics, University College Dublin, Dublin, Ireland

13 Sezione INFN di Bari, Bari, Italy

14 Sezione INFN di Bologna, Bologna, Italy

15 Sezione INFN di Cagliari, Cagliari, Italy

16 Sezione INFN di Ferrara, Ferrara, Italy

17 Sezione INFN di Firenze, Firenze, Italy

18 Laboratori Nazionali dell'INFN di Frascati, Frascati, Italy

19 Sezione INFN di Genova, Genova, Italy

20 Sezione INFN di Milano Bicocca, Milano, Italy

21 Sezione INFN di Padova, Padova, Italy

22 Sezione INFN di Pisa, Pisa, Italy

23 Sezione INFN di Roma Tor Vergata, Roma, Italy

24 Sezione INFN di Roma La Sapienza, Roma, Italy

25 Henryk Niewodniczanski Institute of Nuclear Physics Polish Academy of Sciences, Kraków, Poland

26 AGH - University of Science and Technology, Faculty of Physics and Applied Computer Science, Kraków, Poland

27 National Center for Nuclear Research (NCBJ), Warsaw, Poland

28 Horia Hulubei National Institute of Physics and Nuclear Engineering, Bucharest-Magurele, Romania

29 Petersburg Nuclear Physics Institute (PNPI), Gatchina, Russia

30 Institute of Theoretical and Experimental Physics (ITEP), Moscow, Russia

31 Institute of Nuclear Physics, Moscow State University (SINP MSU), Moscow, Russia

32 Institute for Nuclear Research of the Russian Academy of Sciences (INR RAN), Moscow, Russia

33 Budker Institute of Nuclear Physics (SB RAS) and Novosibirsk State University, Novosibirsk, Russia

34 Institute for High Energy Physics (IHEP), Protvino, Russia 
Universitat de Barcelona, Barcelona, Spain

39 Physik-Institut, Universität Zürich, Zürich, Switzerland

40 Nikhef National Institute for Subatomic Physics, Amsterdam, The Netherlands

41 Nikhef National Institute for Subatomic Physics and VU University Amsterdam, Amsterdam, The Netherlands

42 NSC Kharkiv Institute of Physics and Technology (NSC KIPT), Kharkiv, Ukraine

43 Institute for Nuclear Research of the National Academy of Sciences (KINR), Kyiv, Ukraine

44 University of Birmingham, Birmingham, United Kingdom

${ }^{45}$ H.H. Wills Physics Laboratory, University of Bristol, Bristol, United Kingdom

46 Cavendish Laboratory, University of Cambridge, Cambridge, United Kingdom

47 Department of Physics, University of Warwick, Coventry, United Kingdom

48 STFC Rutherford Appleton Laboratory, Didcot, United Kingdom

49 School of Physics and Astronomy, University of Edinburgh, Edinburgh, United Kingdom

50 School of Physics and Astronomy, University of Glasgow, Glasgow, United Kingdom

51 Oliver Lodge Laboratory, University of Liverpool, Liverpool, United Kingdom

52 Imperial College London, London, United Kingdom

53 School of Physics and Astronomy, University of Manchester, Manchester, United Kingdom

54 Department of Physics, University of Oxford, Oxford, United Kingdom

55 Massachusetts Institute of Technology, Cambridge, MA, United States

56 University of Cincinnati, Cincinnati, OH, United States

57 University of Maryland, College Park, MD, United States

58 Syracuse University, Syracuse, NY, United States

59 Pontifícia Universidade Católica do Rio de Janeiro (PUC-Rio), Rio de Janeiro, Brazil, associated to ${ }^{2}$

${ }^{60}$ Institut für Physik, Universität Rostock, Rostock, Germany, associated to ${ }^{11}$

${ }^{a}$ P.N. Lebedev Physical Institute, Russian Academy of Science (LPI RAS), Moscow, Russia

${ }^{b}$ Università di Bari, Bari, Italy

c Università di Bologna, Bologna, Italy

d Università di Cagliari, Cagliari, Italy

e Università di Ferrara, Ferrara, Italy

${ }^{f}$ Università di Firenze, Firenze, Italy

$g$ Università di Urbino, Urbino, Italy

${ }^{h}$ Università di Modena e Reggio Emilia, Modena, Italy

${ }^{i}$ Università di Genova, Genova, Italy

j Università di Milano Bicocca, Milano, Italy

${ }^{k}$ Università di Roma Tor Vergata, Roma, Italy

$l$ Università di Roma La Sapienza, Roma, Italy

$m$ Università della Basilicata, Potenza, Italy

${ }^{n}$ LIFAELS, La Salle, Universitat Ramon Llull, Barcelona, Spain

o Hanoi University of Science, Hanoi, Viet Nam

$p$ Institute of Physics and Technology, Moscow, Russia

$q$ Università di Padova, Padova, Italy

${ }^{r}$ Università di Pisa, Pisa, Italy

$s$ Scuola Normale Superiore, Pisa, Italy 